\title{
Prevalence of HIV infection among female sex workers in the Eastern Mediterranean Region countries: a systematic review
}

\author{
Leili Abedi ${ }^{1}$, Narges Khanjani ${ }^{2}$, Hamid Sharifi ${ }^{3}$ \\ ${ }^{1}$ Department of Statistics and Epidemiology, Faculty of Health, Kerman University of Medical Sciences, Kerman, Iran \\ ${ }^{2}$ Neurology Research Center, Kerman University of Medical Sciences, Kerman, Iran \\ ${ }^{3}$ HIV/STI Surveillance Research Center, and WHO Collaborating Center for HIV, Institute for Futures Studies in Health, \\ Kerman University of Medical Sciences, Kerman, Iran
}

\begin{abstract}
Female sex workers (FSWs) are one of the groups most affected by human immunodeficiency virus (HIV) infection. In Eastern Mediterranean (EM) countries, HIV prevalence among FSWs is often extremely high. However, no review has been conducted on FSWs in EM countries. The purpose of this systematic review was to estimate the prevalence of HIV infection among FSWs in EM countries.

Articles published on the prevalence of HIV infection among FSWs in EM countries were searched until September 10, 2020. Appropriate keywords were used in Web of Science, Scopus, PubMed, EMBASE, Ovid, and IMEMER databases. References of each paper and conference abstracts were additionally searched. Papers were selected according to inclusion and exclusion criteria.

In total, 21 studies were selected, which were performed in 8 EM countries. The reported prevalence of HIV infection ranged from 0 to $16 \%$ among FSWs. The average time women worked as FSWs ranged from 2 to 6 years, and in some papers, FSWs never or rarely used condoms during sexual contact. However, the consistency of using condoms among FSWs was reported as high as $65 \%$ in Pakistan, 64\% in Lebanon, and 62\% In Iran. The prevalence of drug use was high among FSWs, and it ranged from 5\% in Pakistan and Sudan, to 91\% in Iran and $96.2 \%$ in Egypt. Heterogeneity among the studies and sub-groups was very high, and meta-analysis was not done due to high heterogeneity. The reported prevalence of HIV infection varied differently among female sex workers' populations in the Eastern Mediterranean Region. More studies are needed from different EM countries.
\end{abstract}

HIV AIDS Rev 2021; 20, 4: 235-256

DOI: https://doi.org/10.5114/hivar.2021.111233

Key words: Eastern Mediterranean Region, FSWs (female sex workers), HIV infection, prevalence, review.

\section{Introduction}

Human immunodeficiency virus (HIV) is still an important public health issue, even though three decades has passed since its emergence. Currently, nearly 36.7 million people live with HIV infection around the world. In 2016, approximately

Address for correspondence: Prof. Narges Khanjani, Department of Epidemiology and Biostatistics, School of Public Health, Kerman University of Medical Sciences, Kerman, Iran, phone/fax: +98 034-3132-5102, e-mail: n_khanjani@kmu.ac.ir
2.1 million new cases of HIV infections were identified [1]. According to the World Health Organization (WHO), only 19.5 million patients (52\%) out of 36.7 million HIV-infected individuals are treated with antiretroviral drugs [2]. One way of HIV infection transmission is sexual contact, and female sex workers (FSWs) are among the at-risk groups [3].
International Journal of HIV-Related Problems

HIV \& AIDS R e vi e w 
FSWs are usually marginalized and deprived of many things in their living environments, and are perceived as criminals. Therefore, they are more vulnerable to HIV infection [3]. According to the WHO, FSWs are $13.5 \%$ more likely to be infected with HIV than other women in the age of fertility. In Asia, this probability reaches nearly $30 \%$ [4].

In low- to medium-income countries, the prevalence of HIV infection is estimated to be $12 \%$ among FSWs [5]. In the Eastern Mediterranean (EM) countries in 2016, approximately 230,000 people lived with HIV infection, with 21,000 new cases of HIV infection identified, and the prevalence rate was $0.1 \%$ among adults [2]. A study indicated that the probable world prevalence of HIV infection ranged from $0.3 \%$ in the Middle East and North Africa to $29.3 \%$ in sub-Saharan Africa [6]. In 2012, the prevalence of HIV infection was reported 5\% in the Middle East and North Africa, in which the infection was mainly transmitted by FSWs $[7,8]$. In Morocco, a review study showed that the prevalence of infection was $2 \%$ among FSWs, and FSWs played a major role in the transmission of HIV infection in comparison with intravenous drug addicts and homosexual men [9]. According to previous studies, FSWs are exposed to many risk factors, such as having various sex partners, and not using or rarely using condoms [10]. In Libya, the prevalence of HIV infection among FSWs was $15.7 \%$, and it was mainly due to their high number of sex partners and not using condoms [11].

Studies have reported that the frequency of using condoms is higher among customers who pay for sex than those who do not pay for it. Moreover, the prevalence of HIV infection is higher among FSWs who were intravenous drug addicts compared with those FSWs who used non-intravenous drug $[12,13]$. Such individuals are usually infected by a common syringe or unprotected sex [14].

Many review studies have been conducted on the prevalence of AIDS in different parts of the world [15-17]. However, no review has been performed on FSWs in the EM countries. The aim of this systematic review was to investigate the prevalence of HIV infection among female sex workers in the EM countries.

\section{Material and methods}

\section{Search strategy}

In this review, articles published on the prevalence of HIV infection among FSWs in the Eastern Mediterranean (EM) countries until September 10, 2020, were searched. The information was retrieved from both electronic databases and WHO reports [8]. Appropriate keywords were searched in the Web of Science, Scopus, PubMed, EMBASE, Ovid, Google Scholar, and IMEMER (Index Medicos for Eastern Mediterranean Region) databases.

First, the words "Prevalence", "Incidence", and "Frequency” were searched by using an 'OR' operator. In the second step, "Human Immunodeficiency Virus", "Immunodeficiency", "Virus", "Human", "Human Immunodeficiency Viruses", "AIDS Virus", “AIDS Viruses", "Acquired Immune Deficien- cy Syndrome", and "Acquired Immunodeficiency Syndrome Virus" were searched with 'OR' operators. In the third step, "FSWs" was searched. In the fourth step, the names of 21 Eastern Mediterranean countries were searched through an 'OR' operator. Finally, the searches of previous steps were joined using 'AND' operators to narrow down the results. Additionally, references at the end of each paper were verified. Conferences abstracts were also searched in Scopus, SID, and Embase databases.

\section{Eligibility criteria}

The retrieved papers were evaluated, and duplicate papers were deleted by checking the names of authors, publication year, place of study, and sample size. Then, the titles and abstracts of papers were screened according to the inclusion and exclusion criteria to remove irrelevant papers. Later, the entire texts of remaining papers were evaluated. Table 1 demonstrates the flowchart of papers' selection.

The inclusion criteria for the papers included in this review were studies conducted in EM countries, which reported the prevalence of HIV infection among the population, including FSWs who had sex in return for money, drugs, or goods, and their HIV infection had been diagnosed by laboratory tests. Papers that had reported HIV infections based on self-reports, and review papers were deleted. The texts of the papers were evaluated according to STROBE checklist [18]. The items of STROBE checklist were ranked zero (poor), one (medium), or two (good). The poor-quality papers included articles with a total score of 30 or lower. The medium-quality group consisted of the papers with a score between 31 and 35, and the good-quality group included the papers with a score of 36 or higher.

\section{Classification and analysis of published articles}

Key information was extracted from the included papers. A test of heterogeneity was performed before meta-analysis. In order to test heterogeneity, $\chi^{2}$ test and $I^{2}$ test were used along with Galbraith chart. In the $c^{2}$ test, a small $p$-value indicated a significant difference in the results of included studies. The $I^{2}$ test was introduced by Higgins, and it shows what percentage of differences in study results are due to heterogeneity among the results rather than sampling. Generally, values higher than 50\% showed significant heterogeneity among the studies. We assessed the potential for publication bias by using Egger's bias test for regression asymmetry [19]. The Galbraith chart graphically indicates if the results are homogenous by plotting each study's log transferred effect size divided by its SE ( $Z$ score) on $y$-axis and the inverse of SE on $x$-axis [20]. After observing substantial heterogeneity across the studies, meta-regression was performed to identify potential sources of heterogeneity for years of data collection, and sample size. Sub-group analysis was performed according to years of data collection categories (2010 and before, after 2010), laboratory methods (DBS [dried 


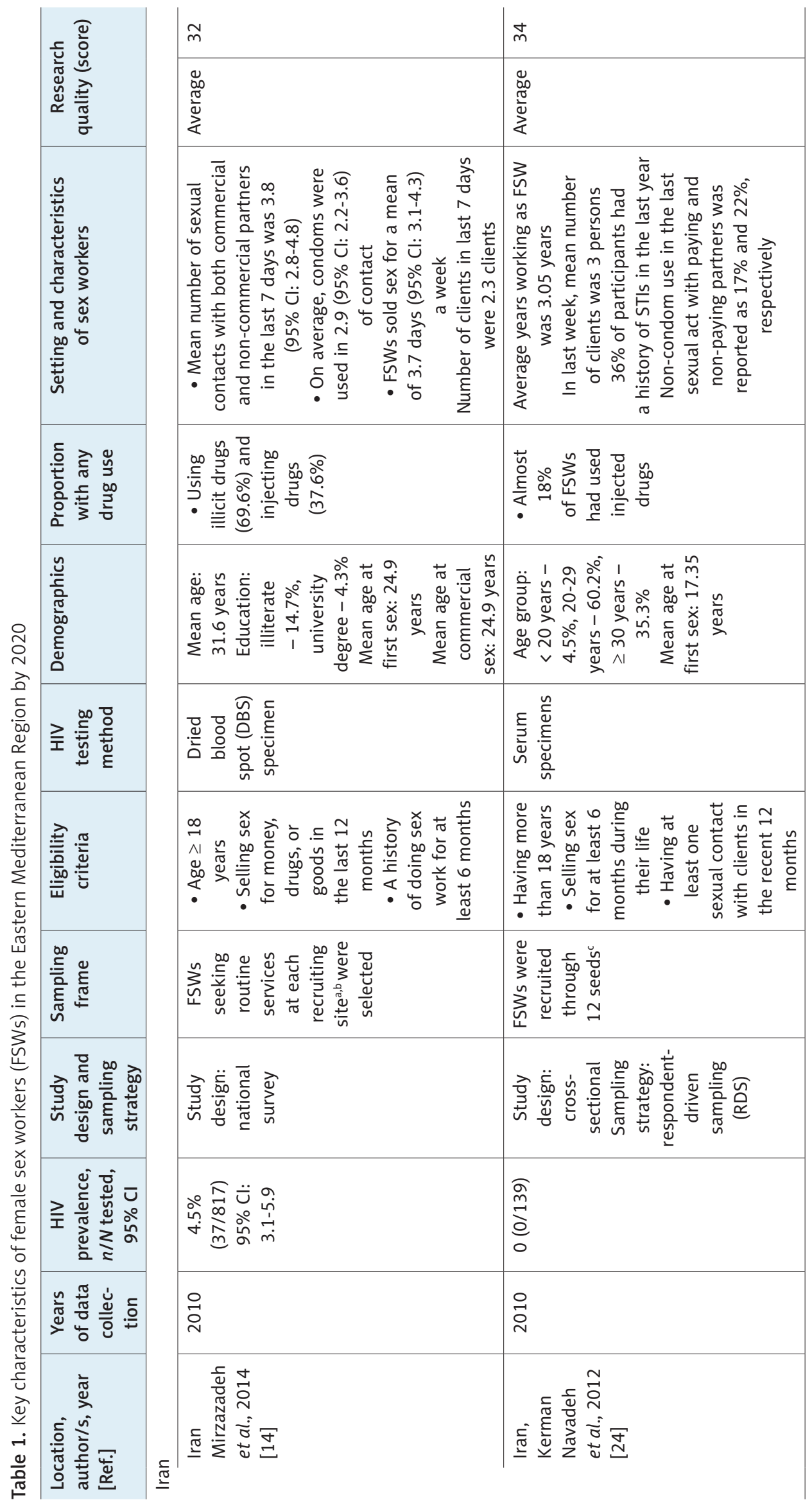




\begin{tabular}{|c|c|c|}
\hline \multirow{2}{*}{ 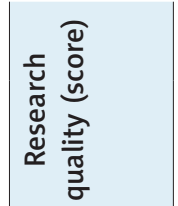 } & $\hat{m}$ & $\stackrel{m}{m}$ \\
\hline & 잉 & 명 \\
\hline 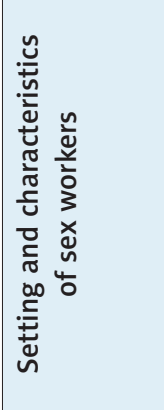 & 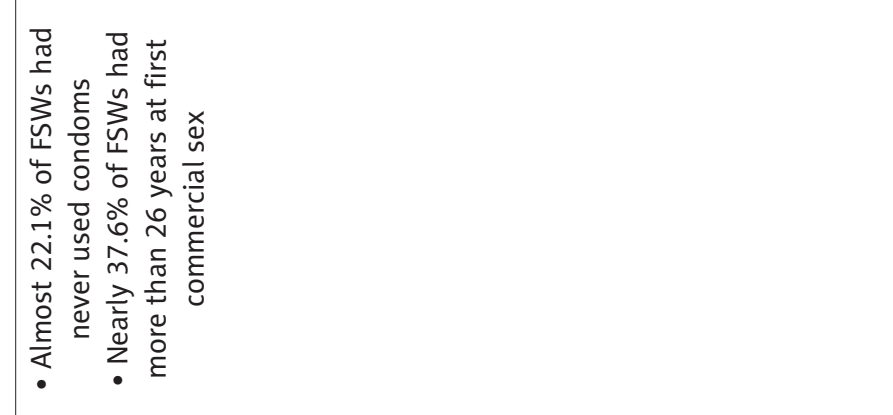 & 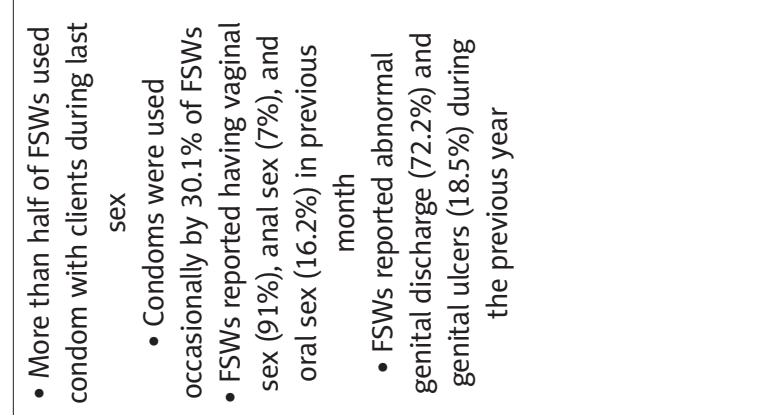 \\
\hline 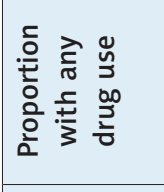 & 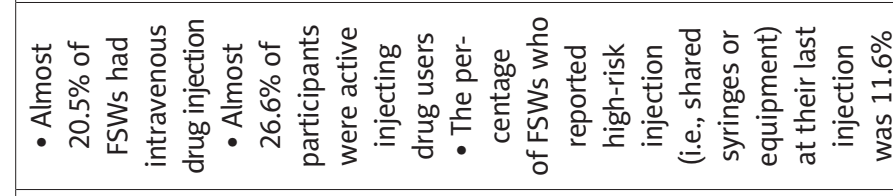 & 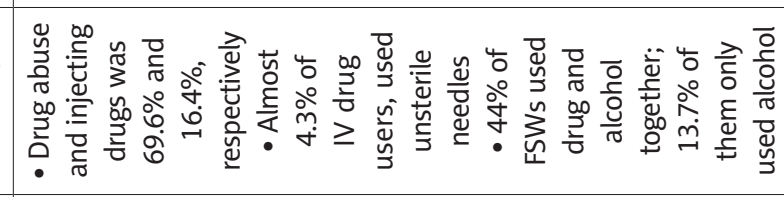 \\
\hline 它 & 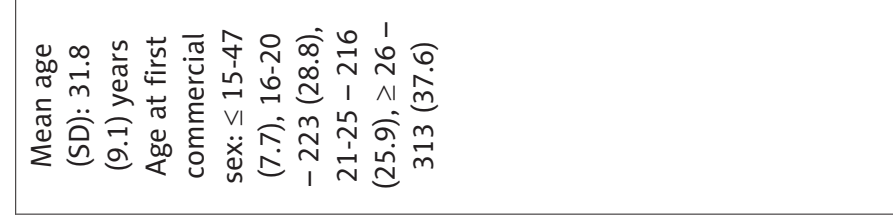 & 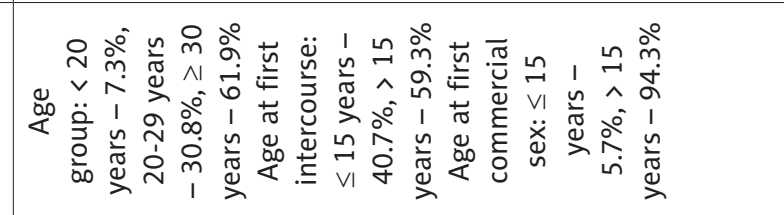 \\
\hline 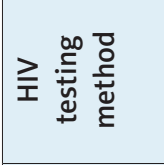 & 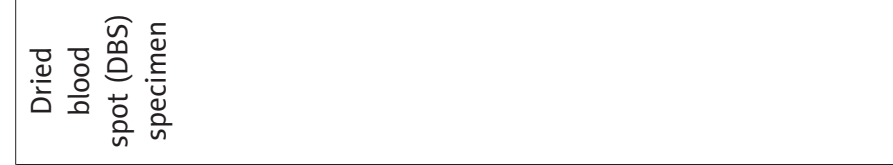 & 䈉 \\
\hline 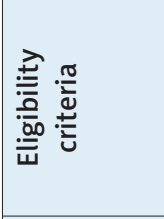 & 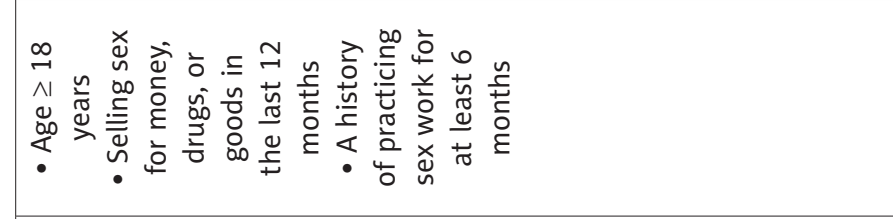 & 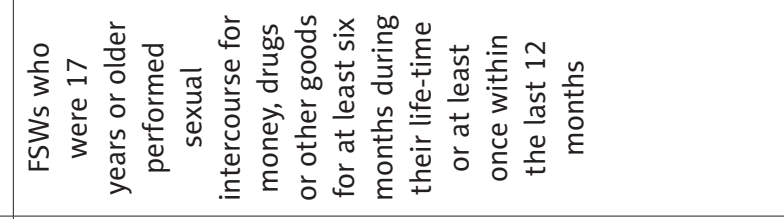 \\
\hline 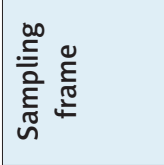 & 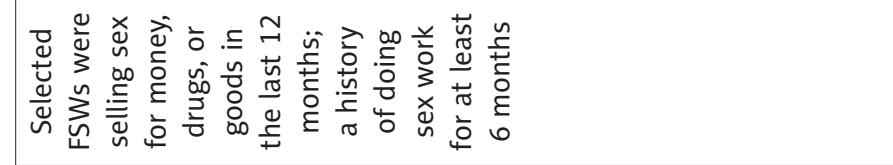 & 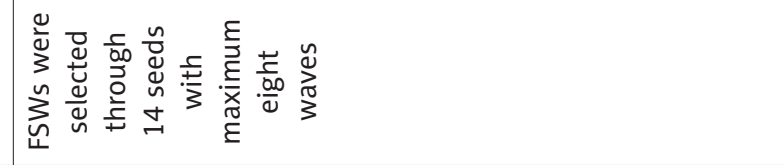 \\
\hline 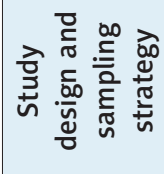 & 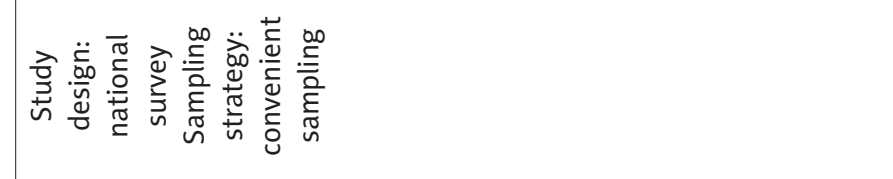 & 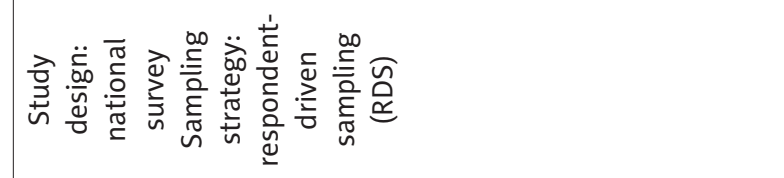 \\
\hline 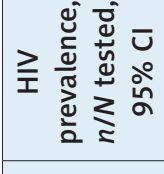 & 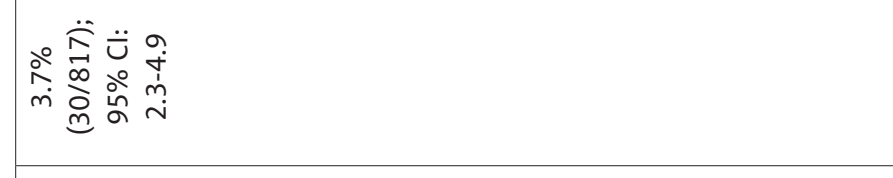 & 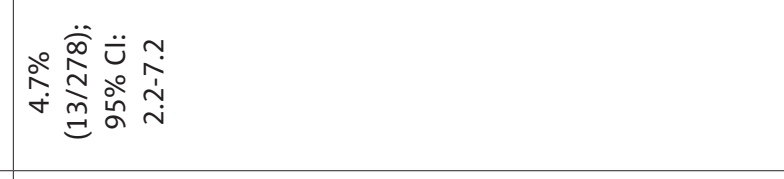 \\
\hline 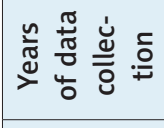 & 옴 & 穴 \\
\hline 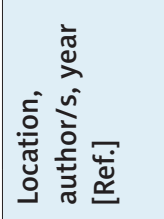 & 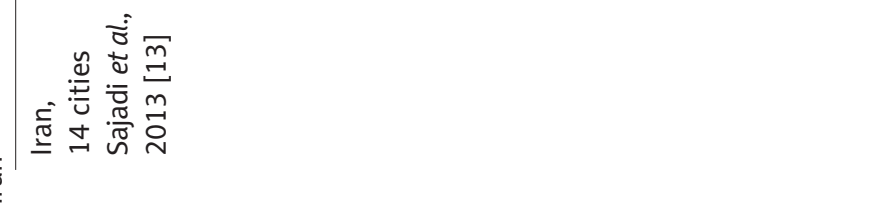 & 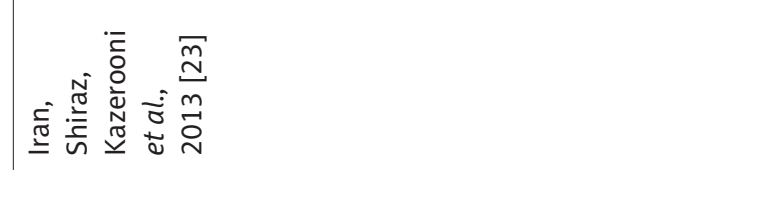 \\
\hline
\end{tabular}




\begin{tabular}{|c|c|}
\hline$=\frac{\bar{v}}{0}$ & N \\
\hline 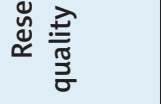 & $\stackrel{\overrightarrow{\mathscr{D}}}{\mathscr{D}^{\circ}}$ \\
\hline 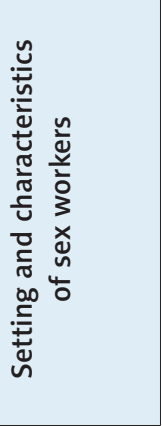 & 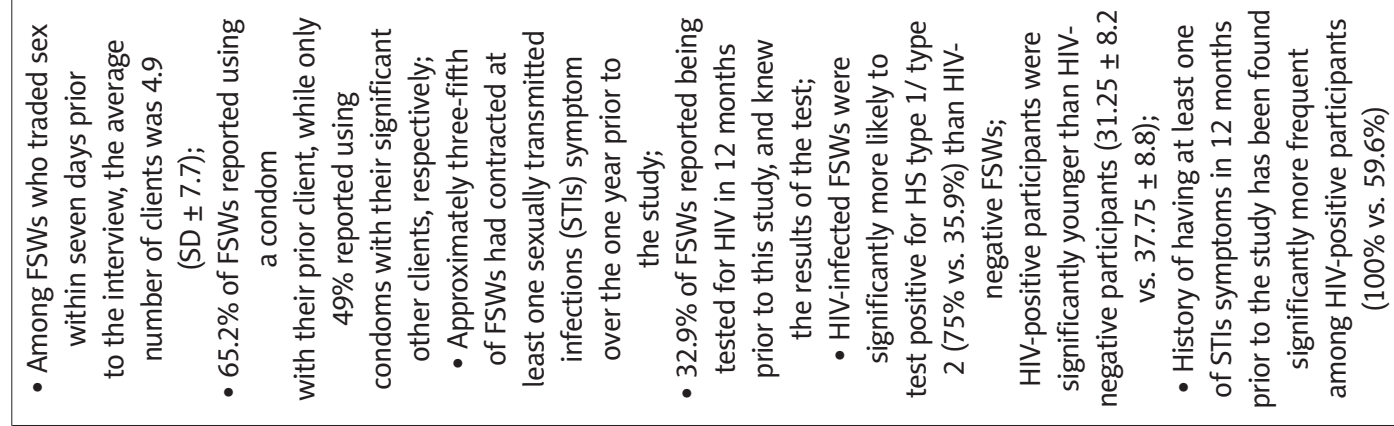 \\
\hline 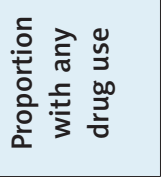 & 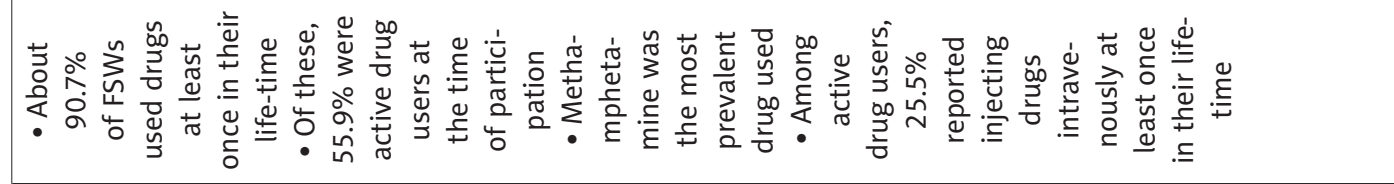 \\
\hline 党 & 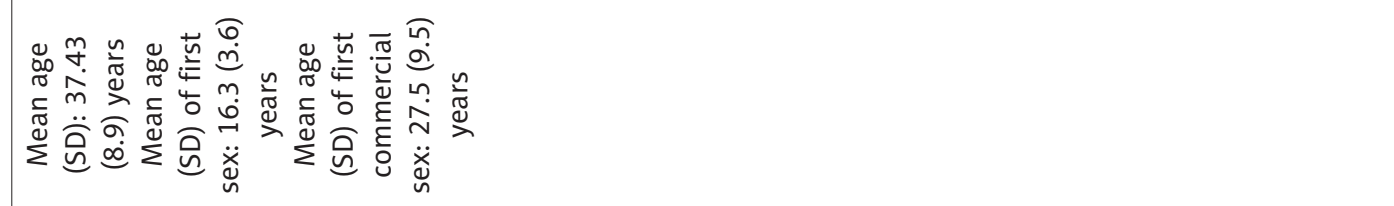 \\
\hline 引 & 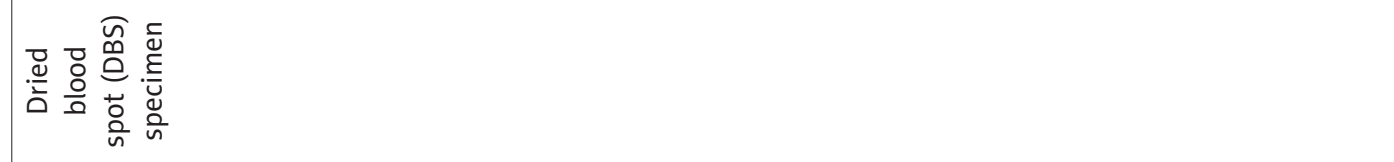 \\
\hline 謩 & 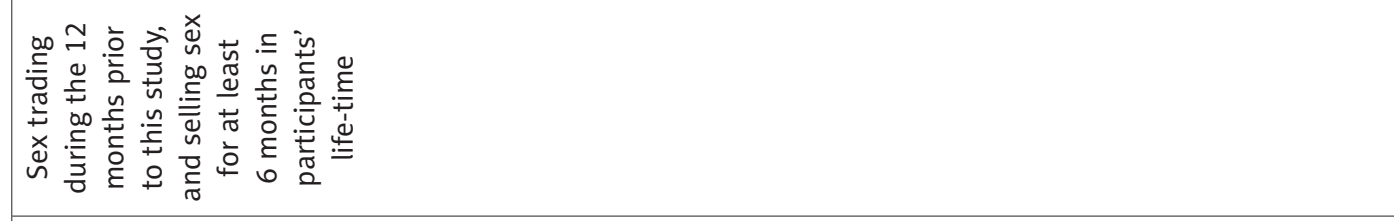 \\
\hline 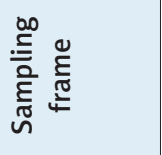 & 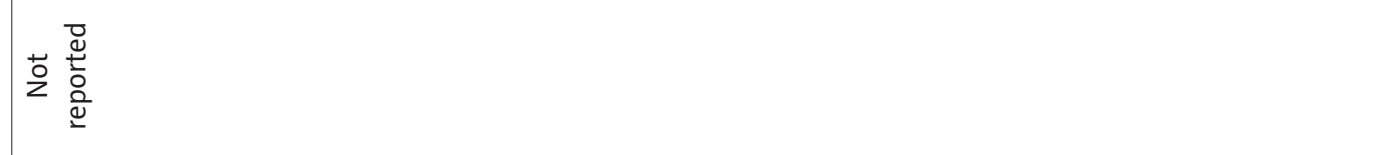 \\
\hline 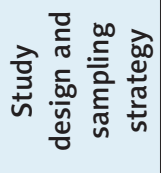 & 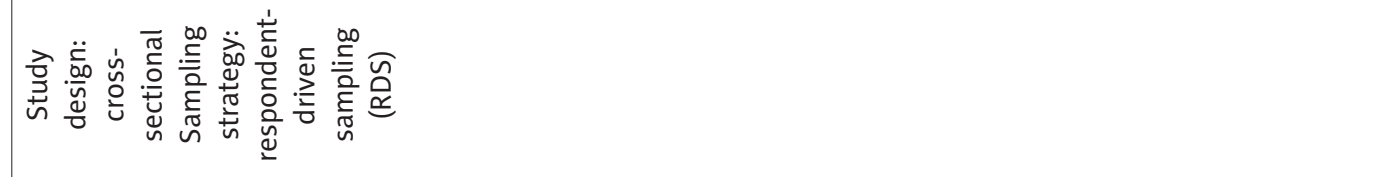 \\
\hline 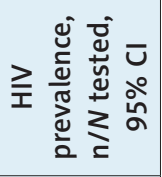 & 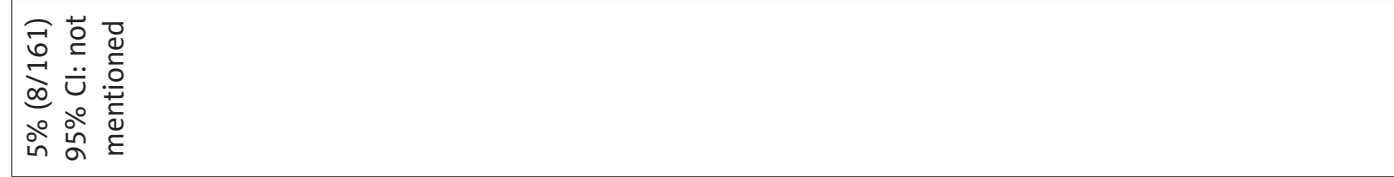 \\
\hline 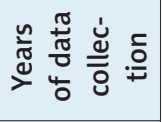 & 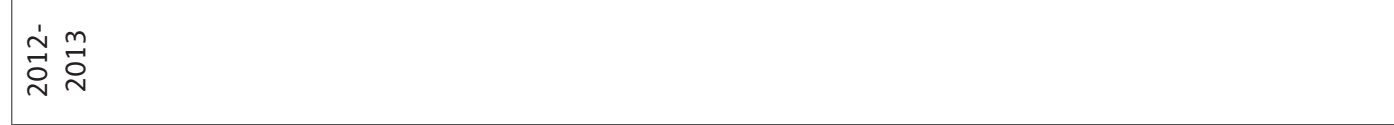 \\
\hline 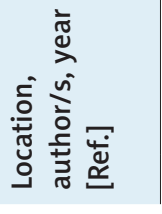 & 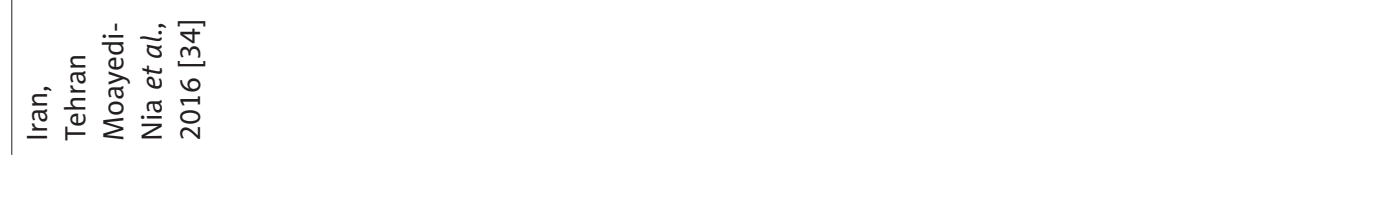 \\
\hline
\end{tabular}




\begin{tabular}{|c|c|}
\hline \multirow{2}{*}{ 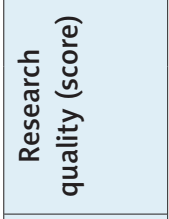 } & $\tilde{m}$ \\
\hline & 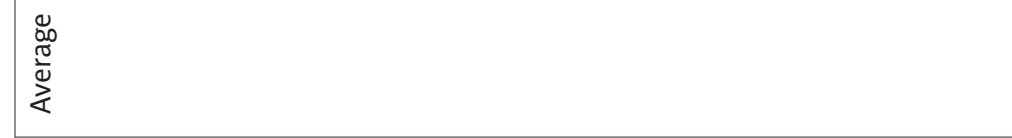 \\
\hline 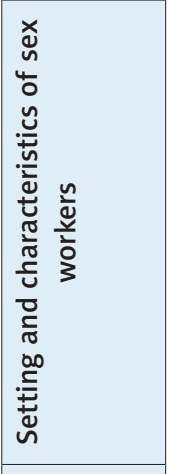 & 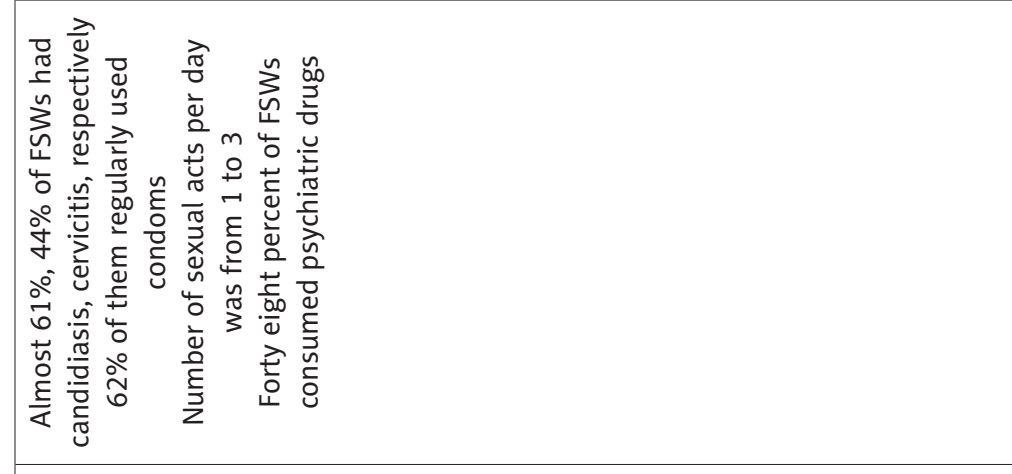 \\
\hline 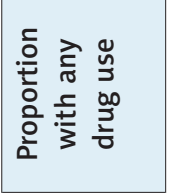 & 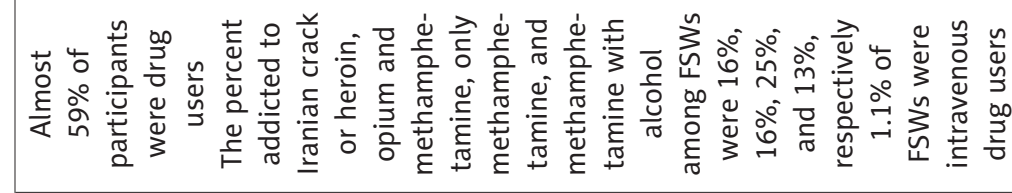 \\
\hline 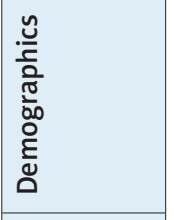 & 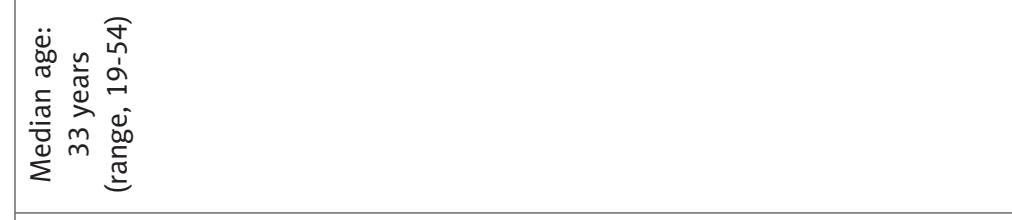 \\
\hline 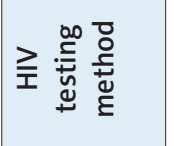 & 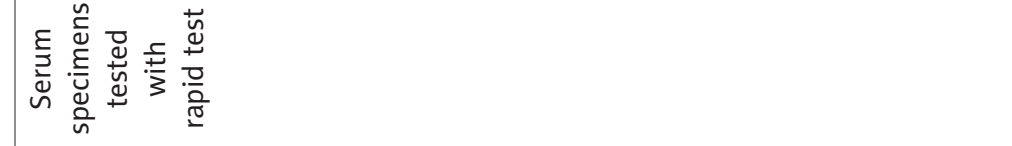 \\
\hline 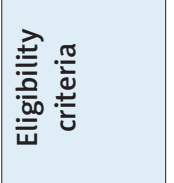 & 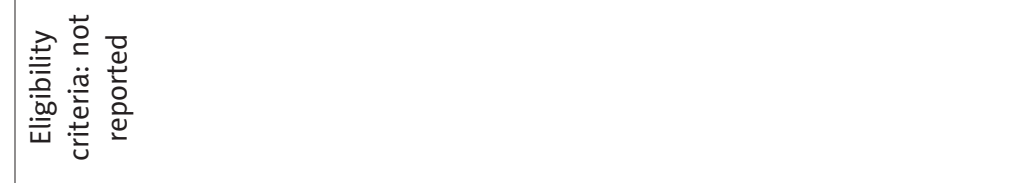 \\
\hline 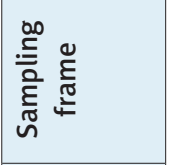 & 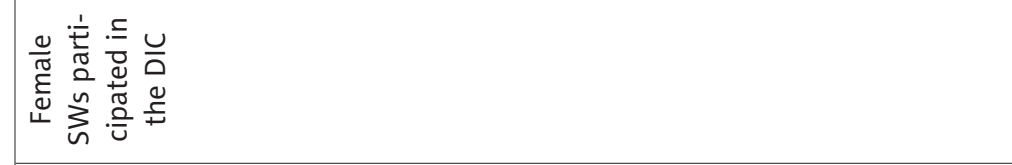 \\
\hline 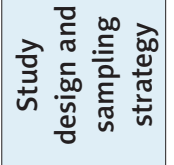 & 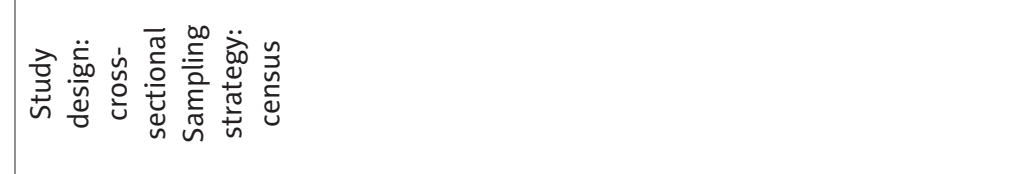 \\
\hline 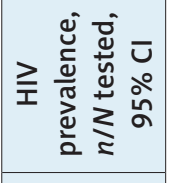 & 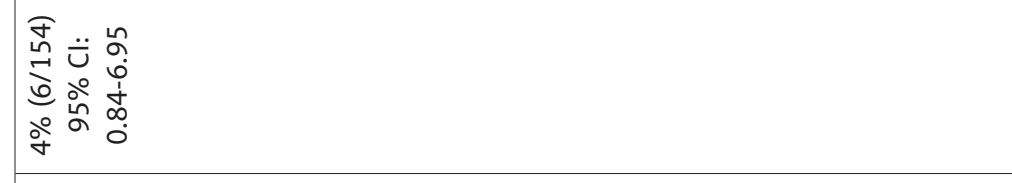 \\
\hline 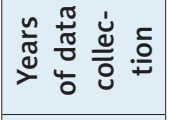 & $\stackrel{+}{\stackrel{N}{N}}$ \\
\hline 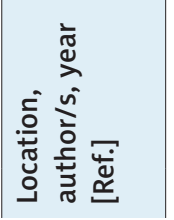 & 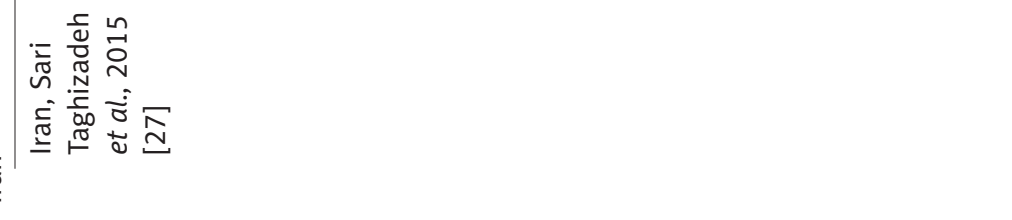 \\
\hline
\end{tabular}




\begin{tabular}{|c|c|}
\hline \multirow{2}{*}{ 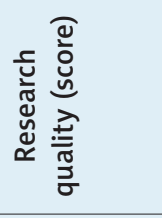 } & $\stackrel{\circ}{m}$ \\
\hline & $\begin{array}{l}\mathbb{D} \\
\infty\end{array}$ \\
\hline 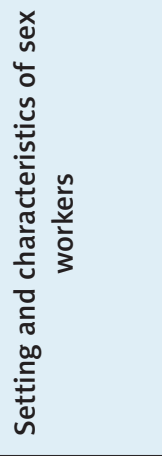 & 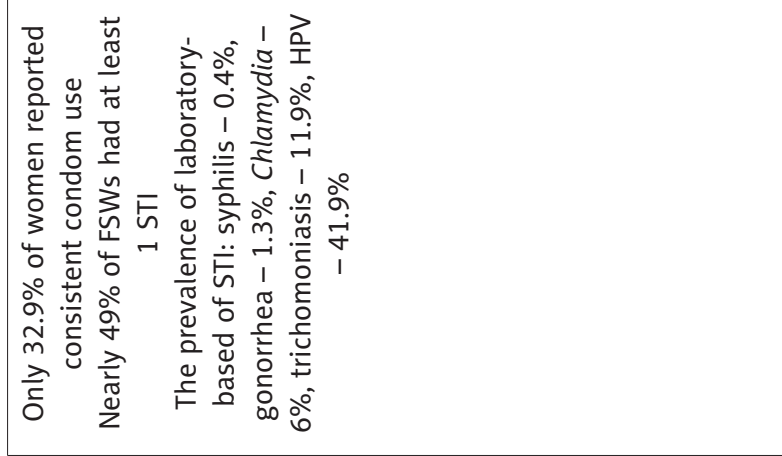 \\
\hline 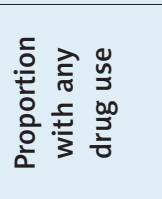 & 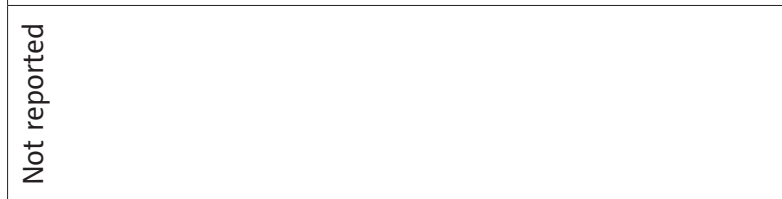 \\
\hline 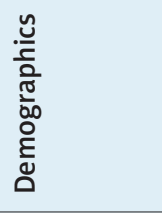 & 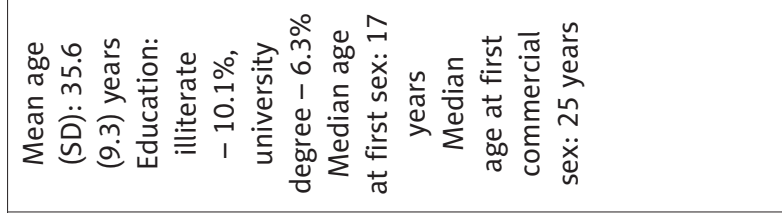 \\
\hline 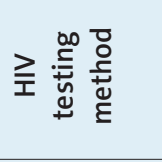 & 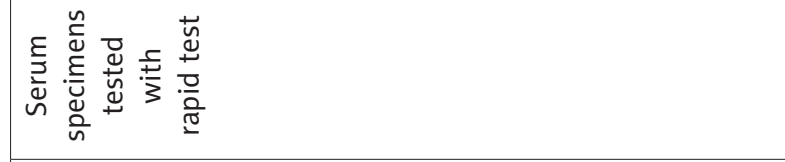 \\
\hline 㕍 & 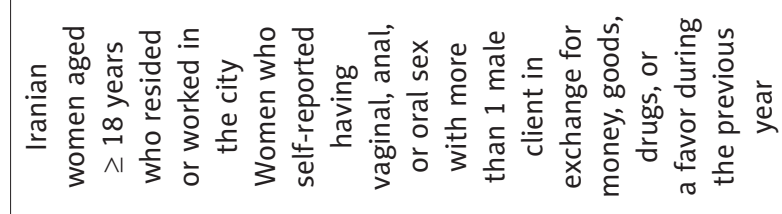 \\
\hline 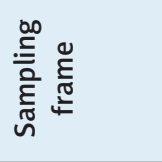 & 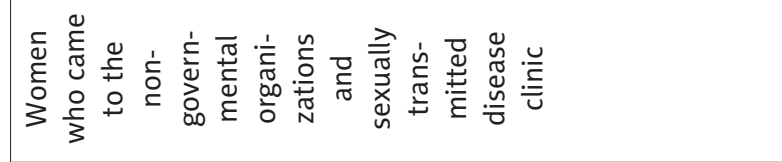 \\
\hline 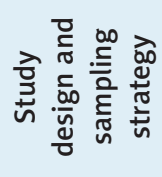 & 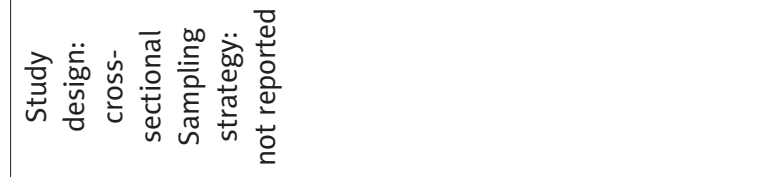 \\
\hline 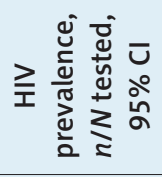 & 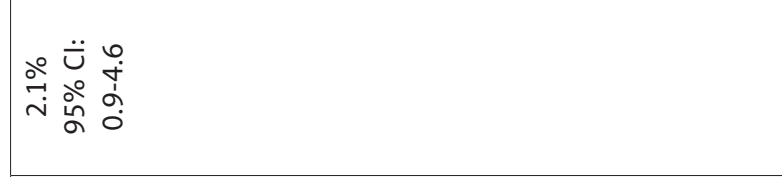 \\
\hline 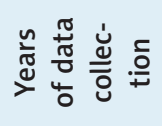 & $\stackrel{\text { na }}{\text { d }}$ \\
\hline 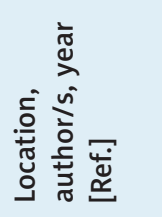 & 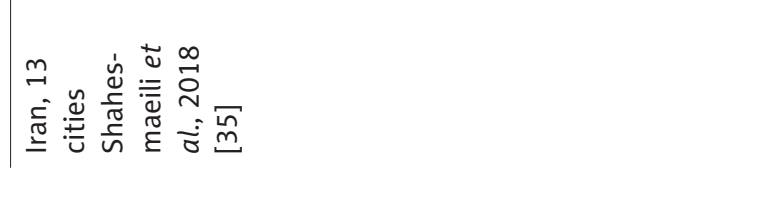 \\
\hline
\end{tabular}




\begin{tabular}{|c|c|c|}
\hline \multirow{2}{*}{ 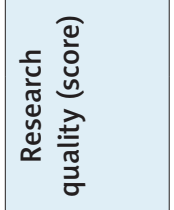 } & $\vec{m}$ & 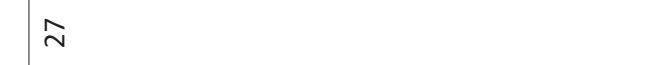 \\
\hline & 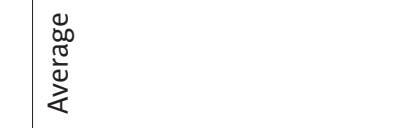 & 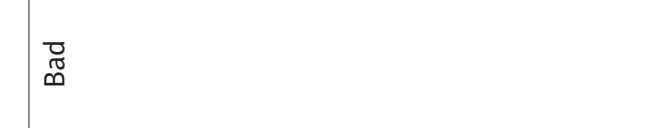 \\
\hline 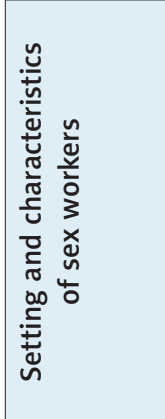 & 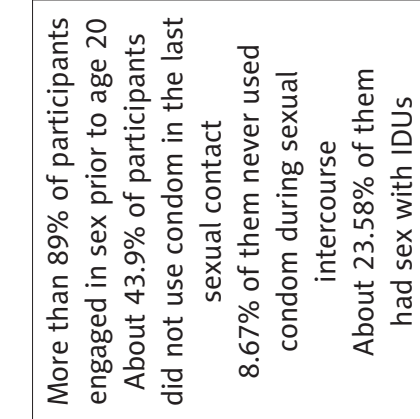 & 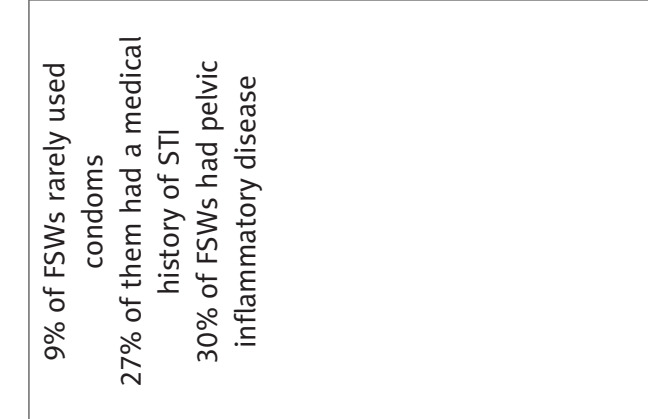 \\
\hline 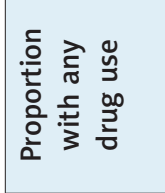 & 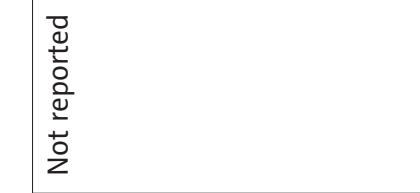 & 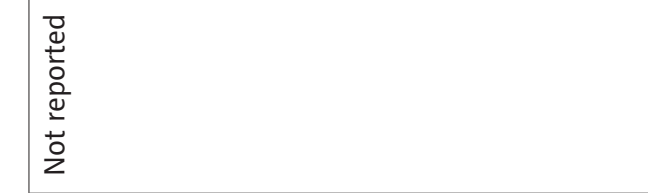 \\
\hline 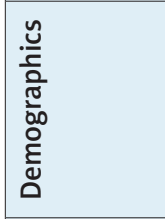 & 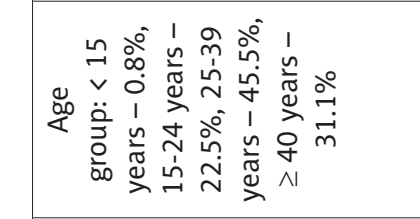 & 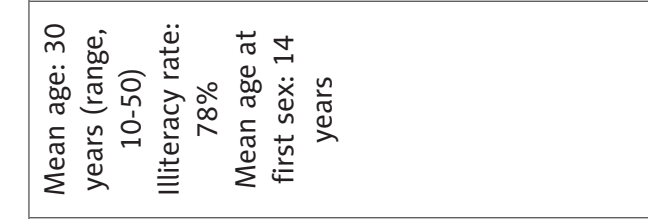 \\
\hline 》立咅总 & 蒙 总 & 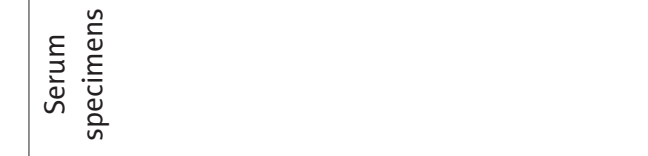 \\
\hline 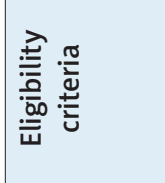 & 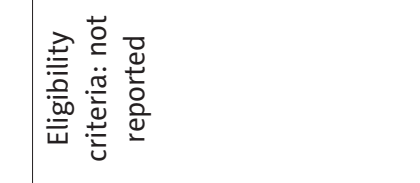 & 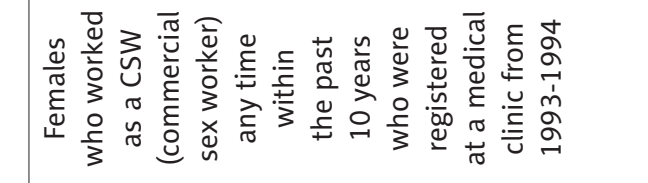 \\
\hline 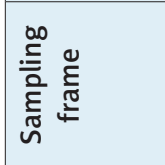 & 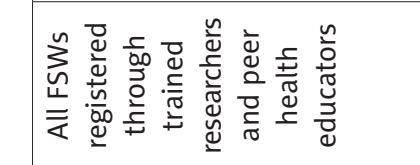 & 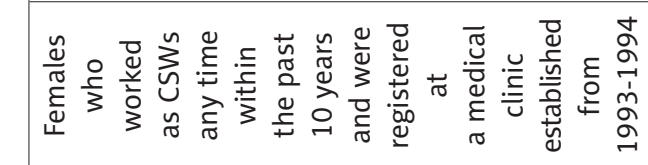 \\
\hline 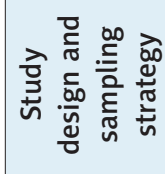 & 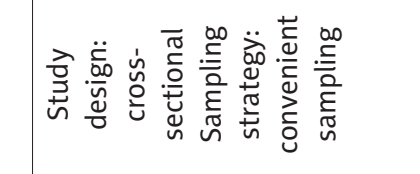 & 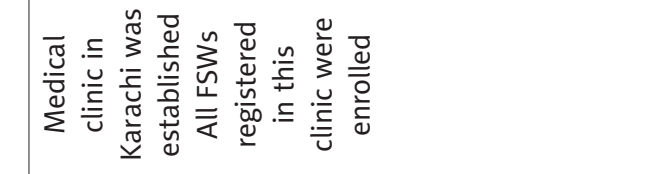 \\
\hline 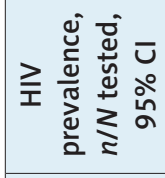 & 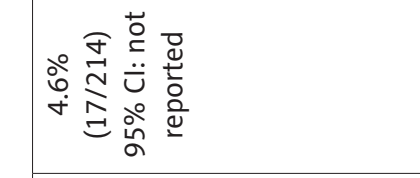 & 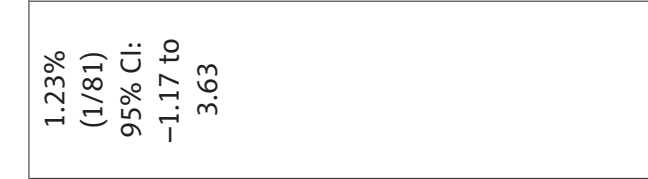 \\
\hline 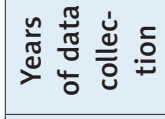 & $\stackrel{\circ}{i}$ & مُ \\
\hline 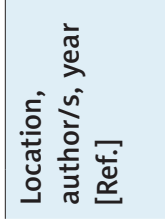 & 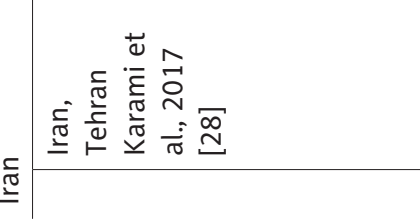 & 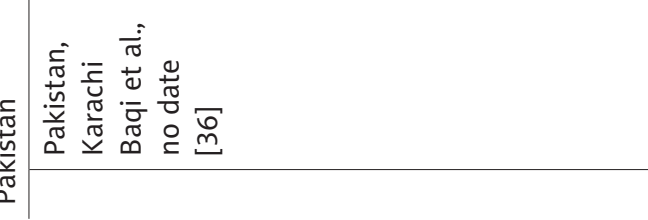 \\
\hline
\end{tabular}




\begin{tabular}{|c|c|c|}
\hline \multirow{2}{*}{ 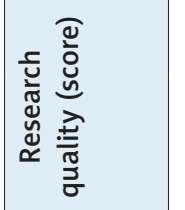 } & $\stackrel{\infty}{\sim}$ & $\stackrel{\sim}{m}$ \\
\hline & $\begin{array}{l}\mathbb{Z} \\
\mathbb{\Phi}\end{array}$ & 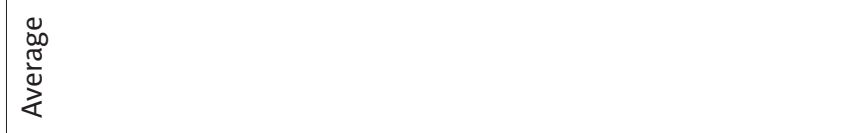 \\
\hline 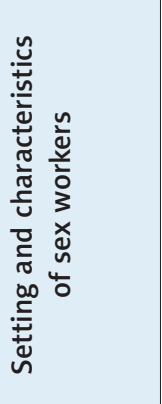 & 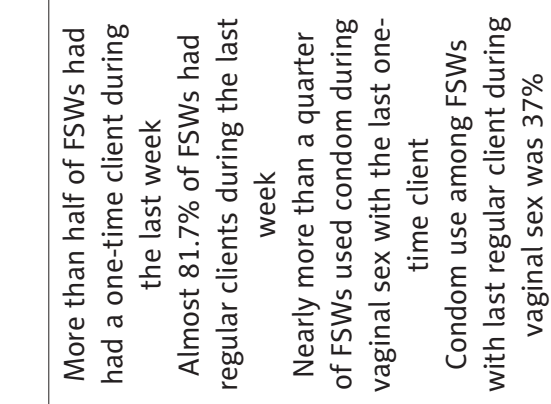 & 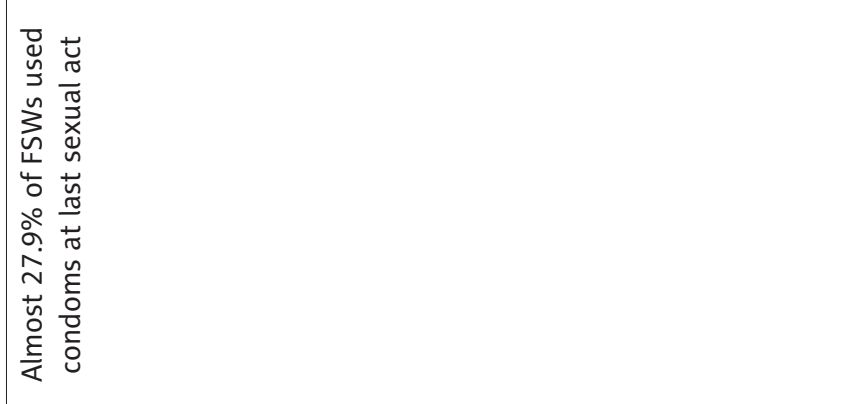 \\
\hline 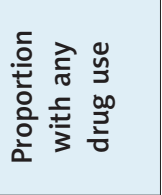 & 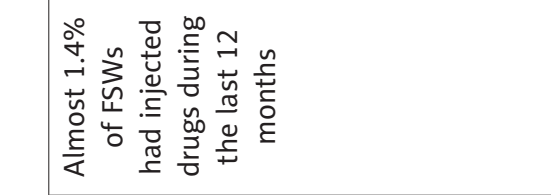 & 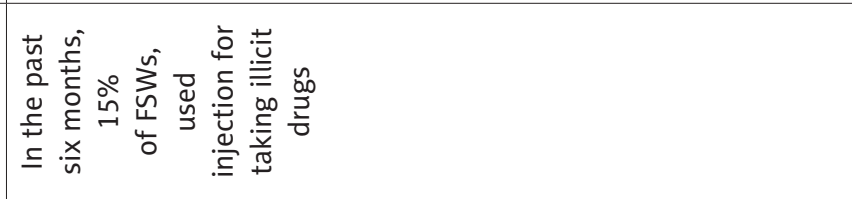 \\
\hline 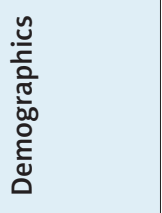 & 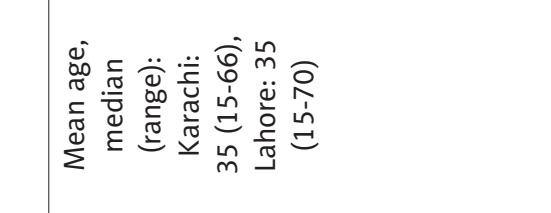 & 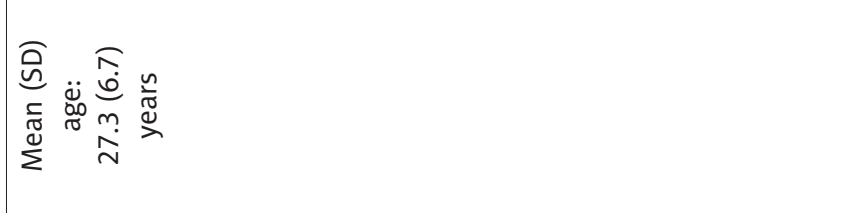 \\
\hline 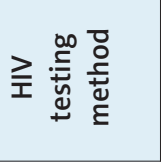 & 音 & 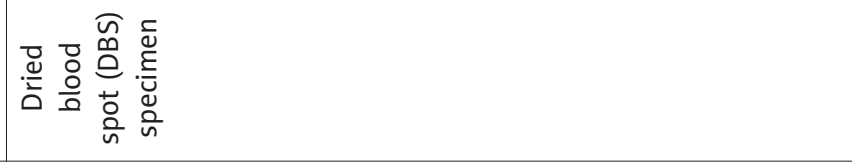 \\
\hline 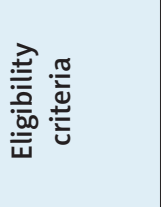 & 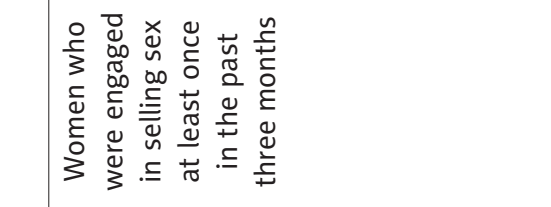 & 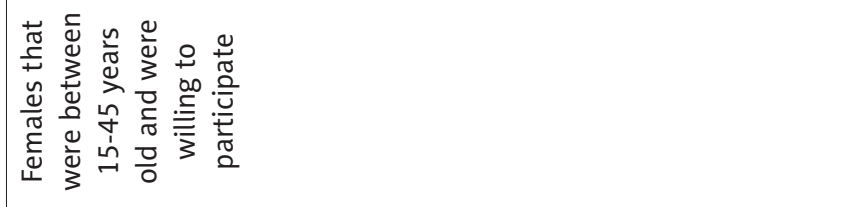 \\
\hline 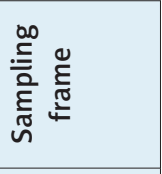 & 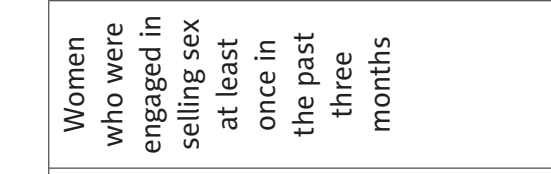 & 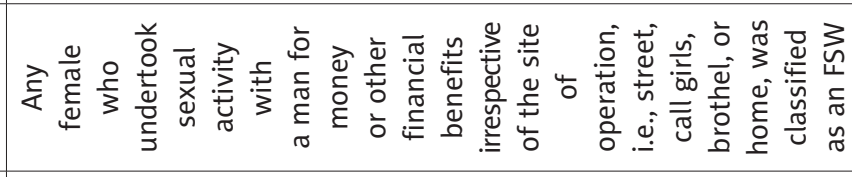 \\
\hline 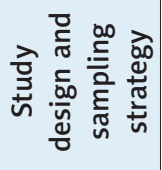 & 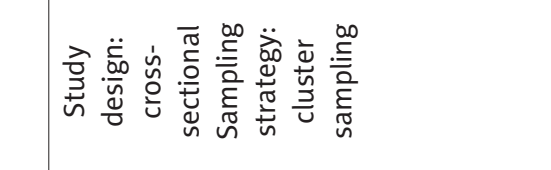 & 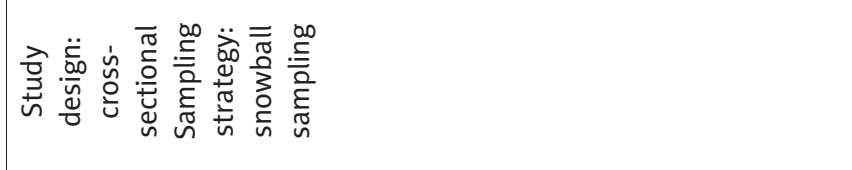 \\
\hline 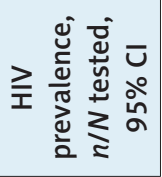 & 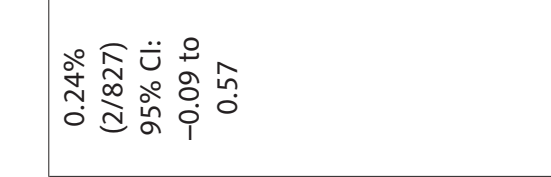 & 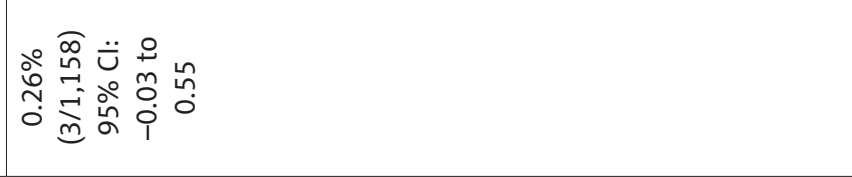 \\
\hline 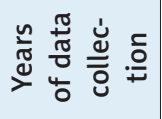 & ষ্ণ & 客 ठํํ \\
\hline 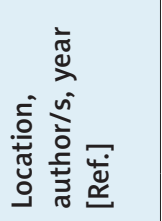 & 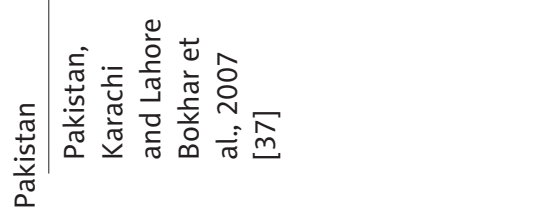 & 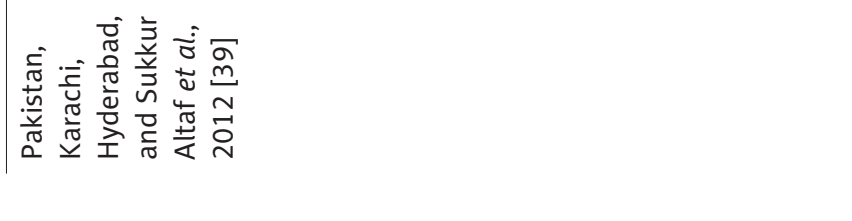 \\
\hline
\end{tabular}




\begin{tabular}{|c|c|c|}
\hline \multirow{2}{*}{ 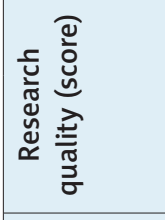 } & $\stackrel{\infty}{m}$ & m \\
\hline & 형 & 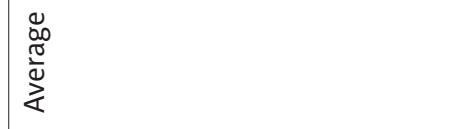 \\
\hline 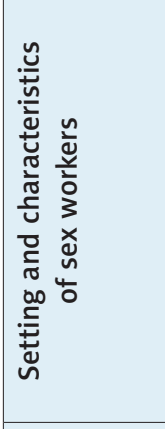 & 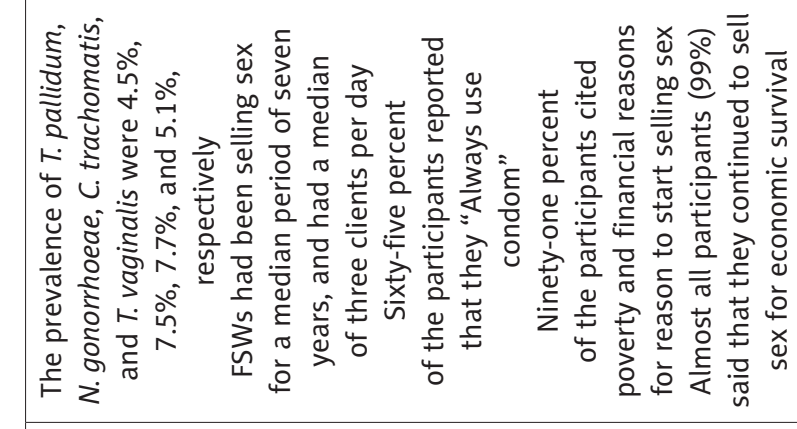 & 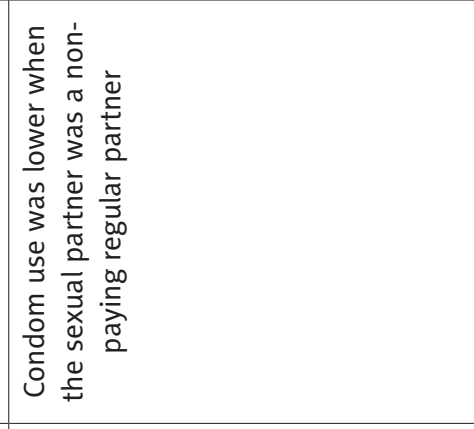 \\
\hline 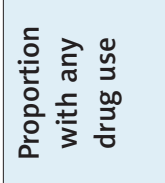 & 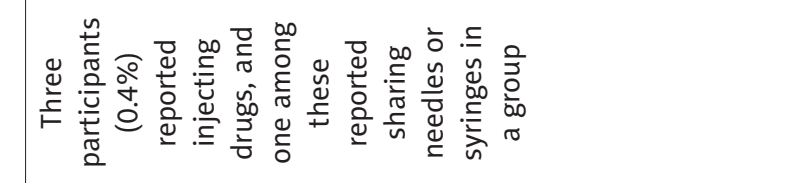 & 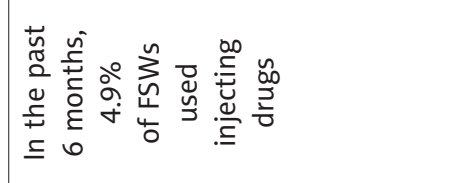 \\
\hline 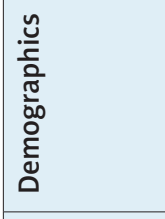 & 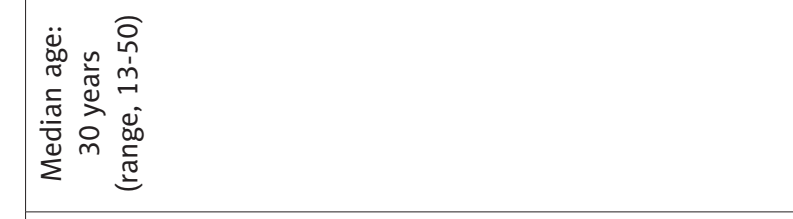 & 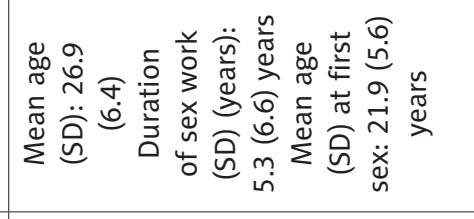 \\
\hline 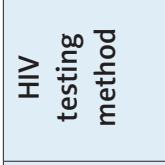 & 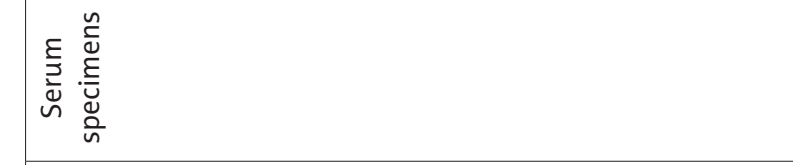 & 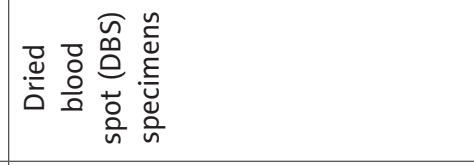 \\
\hline 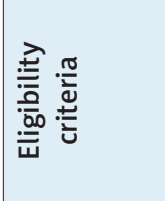 & 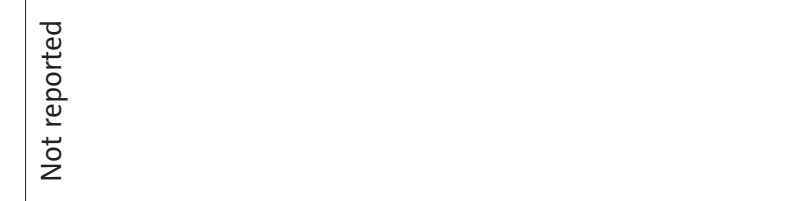 & 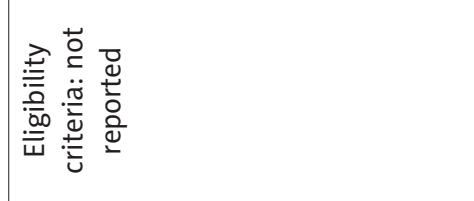 \\
\hline 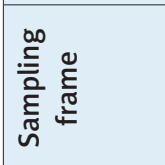 & 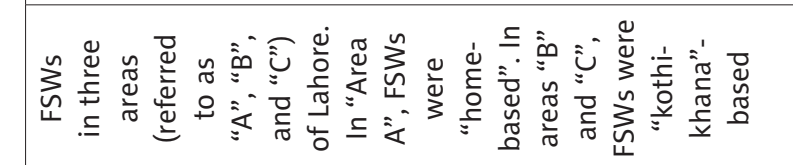 & 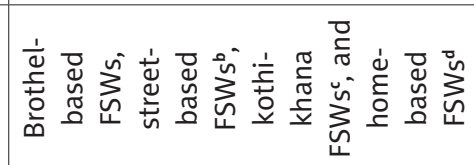 \\
\hline 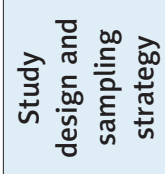 & 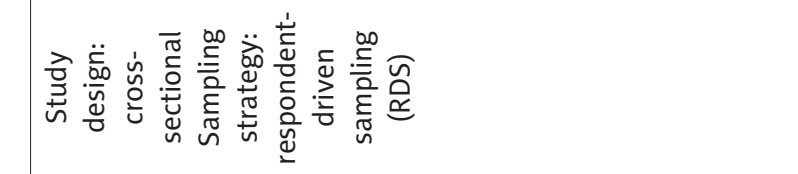 & 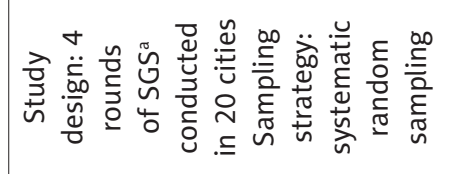 \\
\hline 害离离 & 号递 & 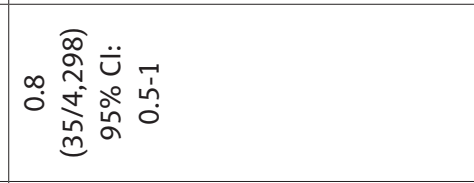 \\
\hline 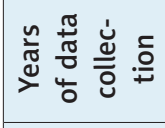 & ঐे & $\dot{\vec{\partial}} \stackrel{\sim}{a}$ \\
\hline 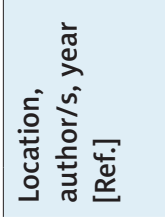 & 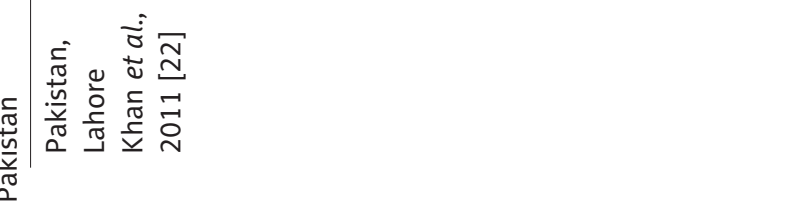 & 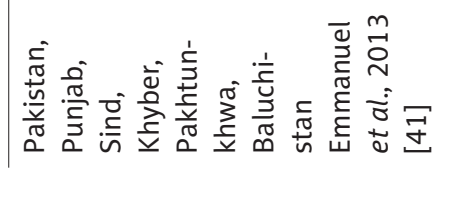 \\
\hline
\end{tabular}




\begin{tabular}{|c|c|c|c|}
\hline \multirow{2}{*}{ 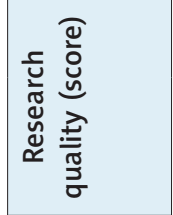 } & $\stackrel{m}{\circ}$ & & \\
\hline & $\begin{array}{l}\mathscr{\Xi} \\
\infty\end{array}$ & 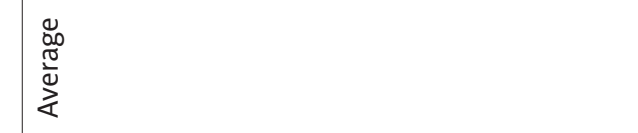 & ठ․ \\
\hline 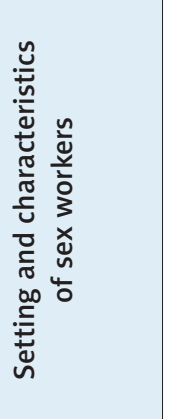 & 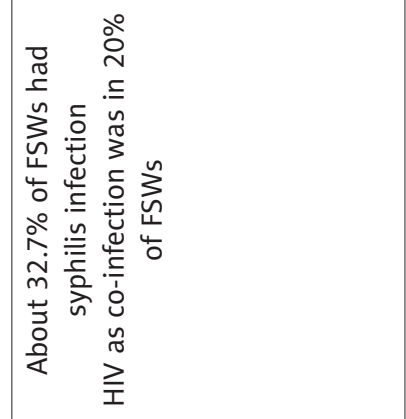 & 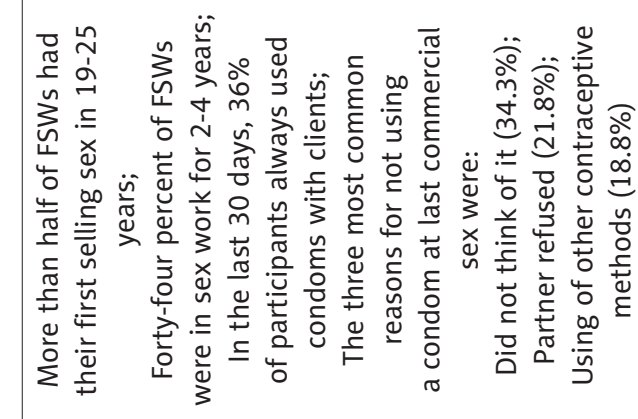 & 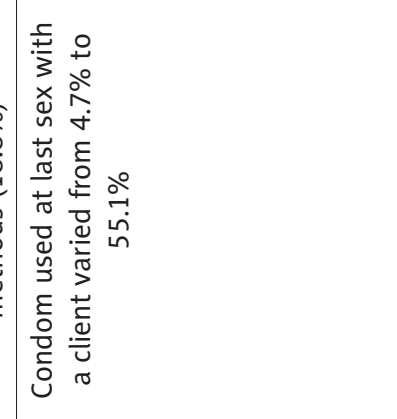 \\
\hline 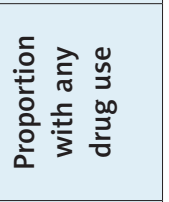 & 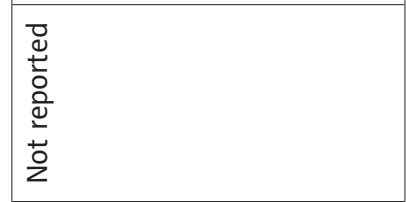 & 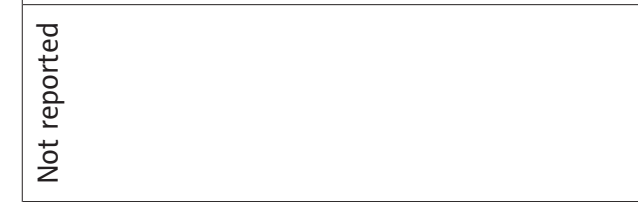 & 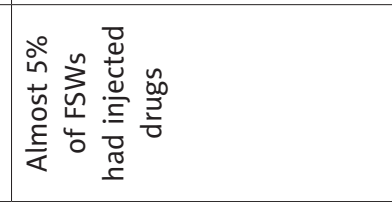 \\
\hline 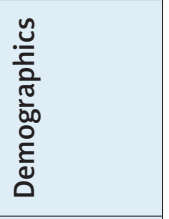 & 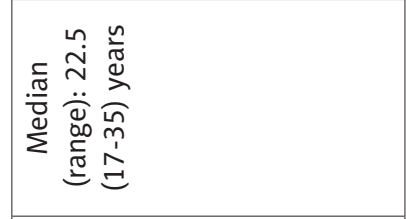 & 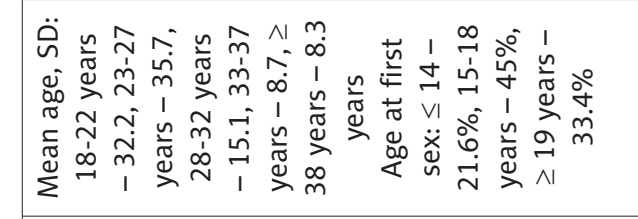 & 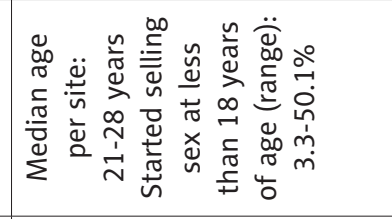 \\
\hline 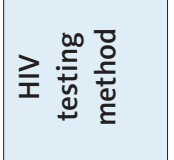 & 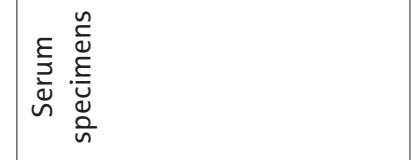 & 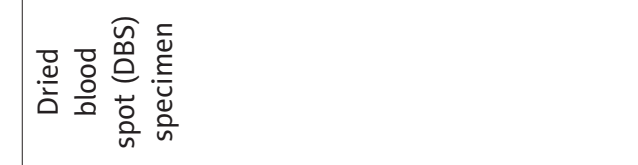 & 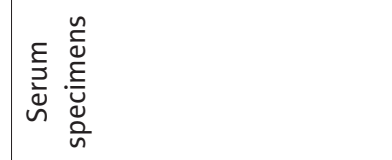 \\
\hline 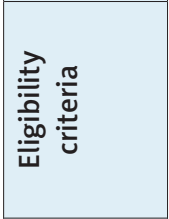 & 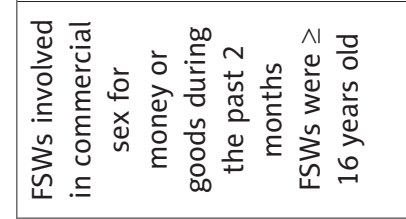 & 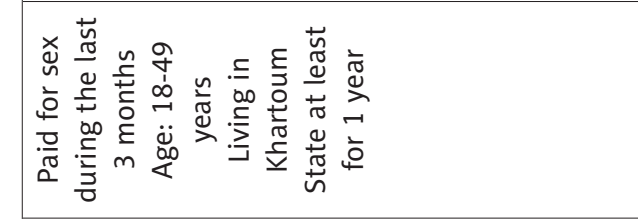 & 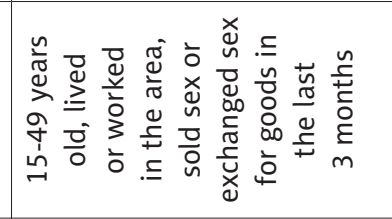 \\
\hline 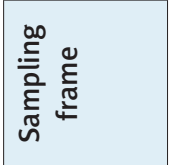 & 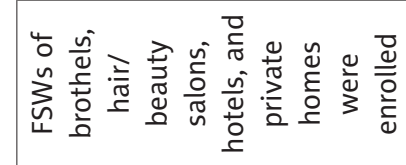 & 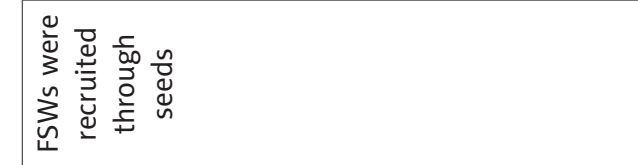 & 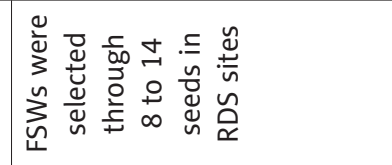 \\
\hline 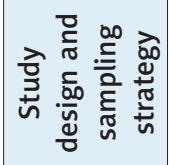 & 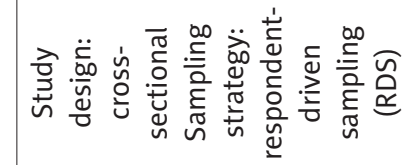 & 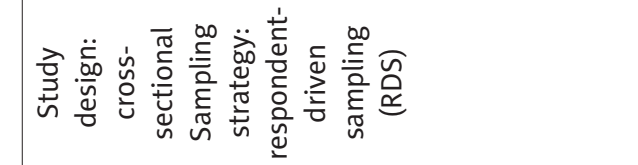 & 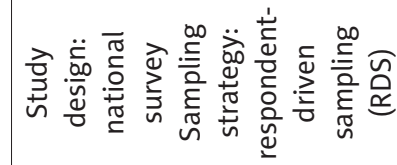 \\
\hline 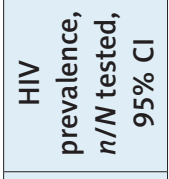 & 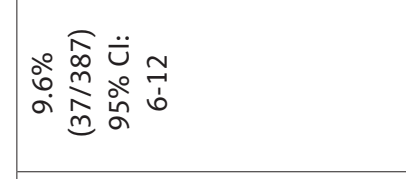 & 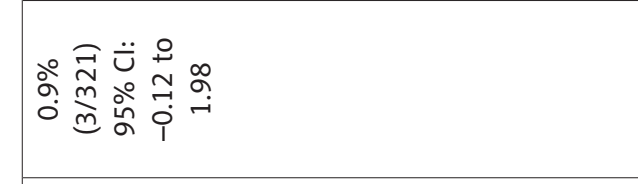 & 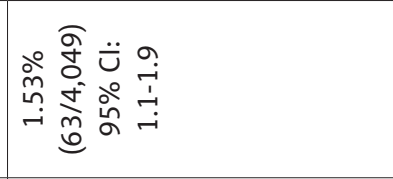 \\
\hline 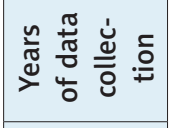 & 空 & 㐫 & $\dot{\vec{\sigma}} \stackrel{\sim}{\sim}$ \\
\hline 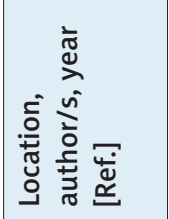 & 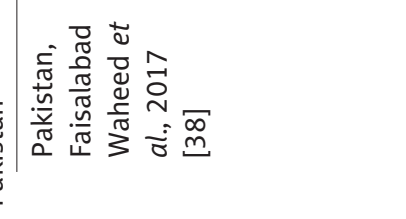 & 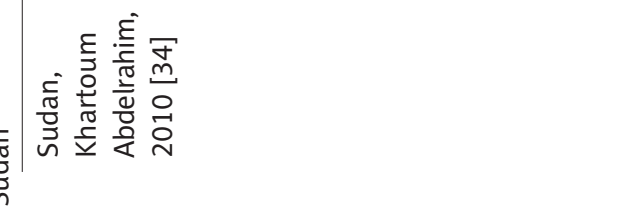 & 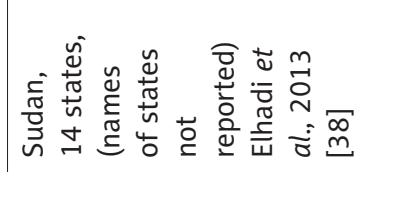 \\
\hline
\end{tabular}




\begin{tabular}{|c|c|c|}
\hline \multirow{2}{*}{ 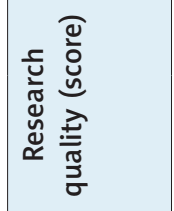 } & $\hat{m}$ & İ \\
\hline & ర্ & 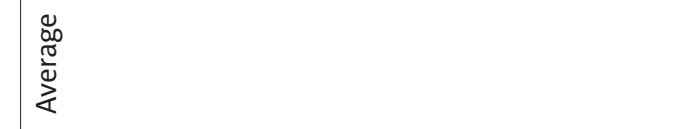 \\
\hline 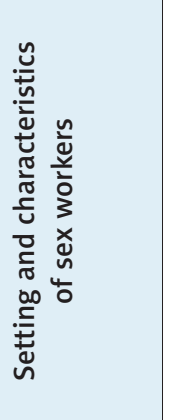 & 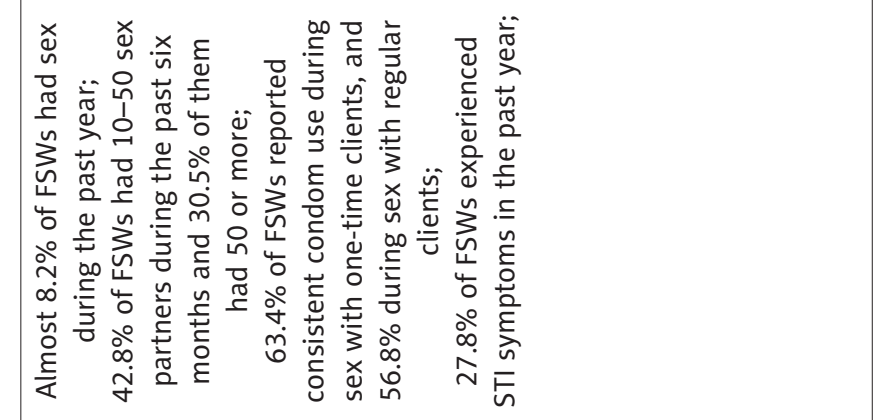 & 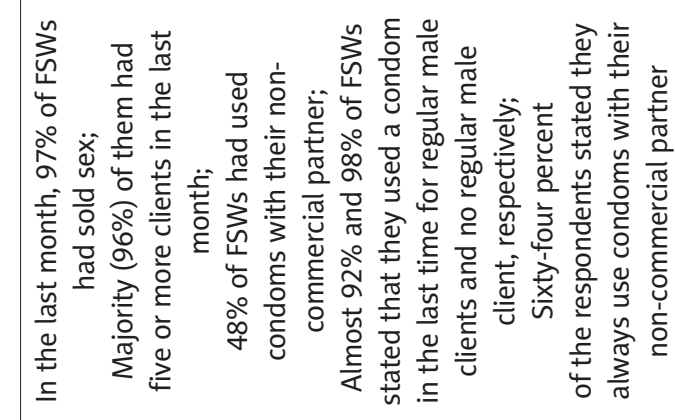 \\
\hline 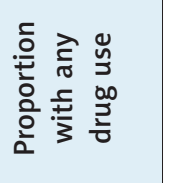 & 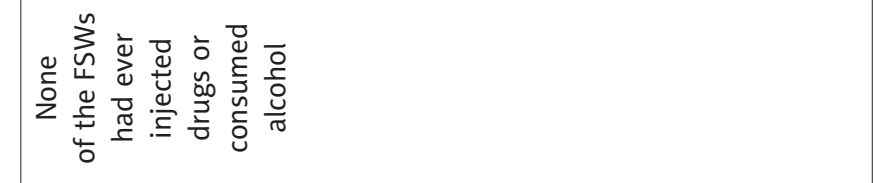 & 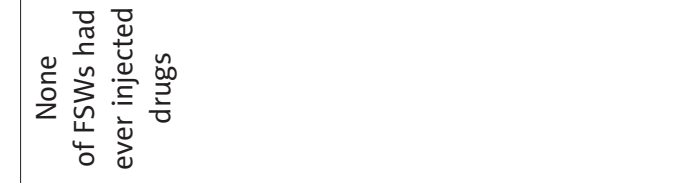 \\
\hline 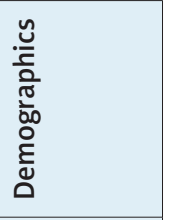 & 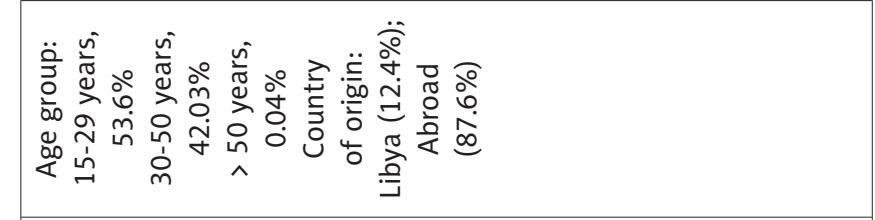 & 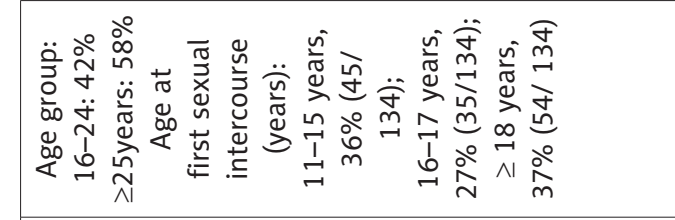 \\
\hline 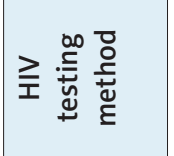 & 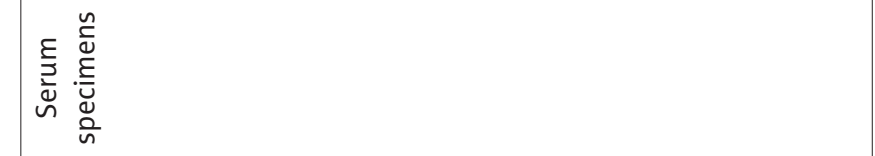 & 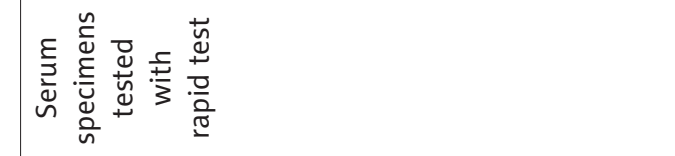 \\
\hline 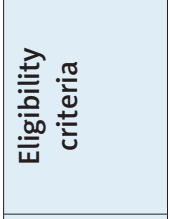 & 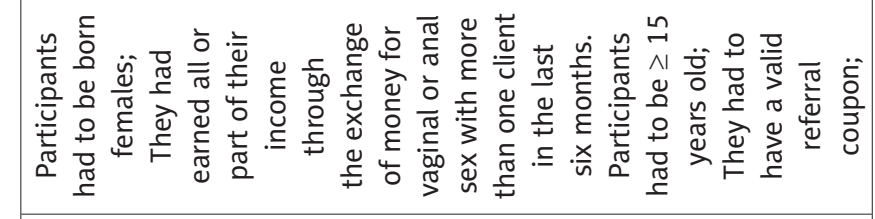 & 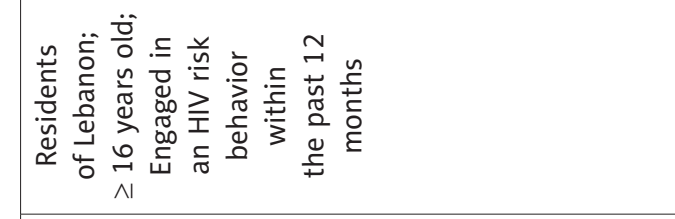 \\
\hline 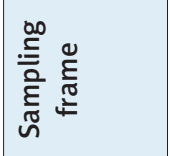 & 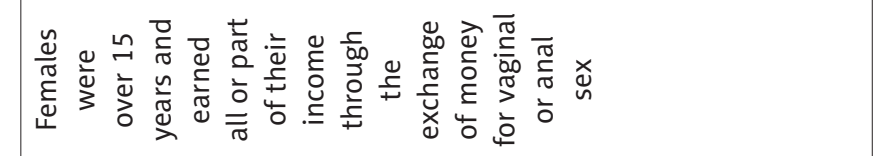 & 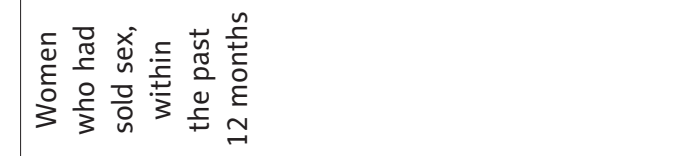 \\
\hline 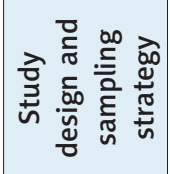 & 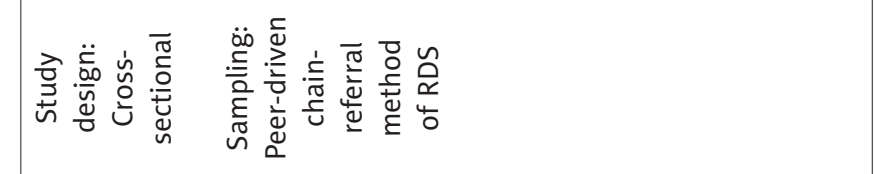 & 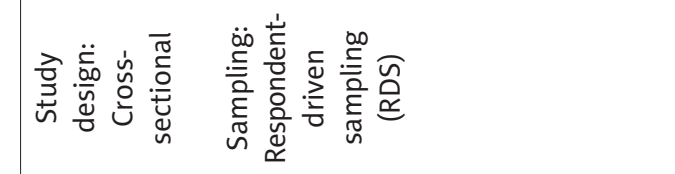 \\
\hline 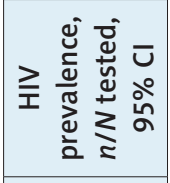 & 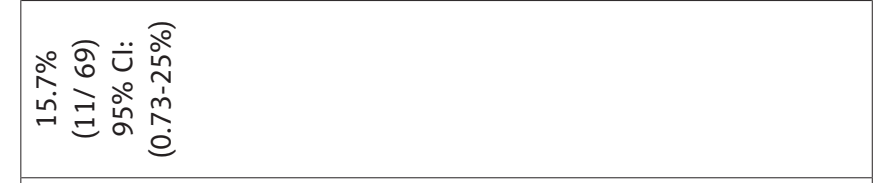 & $\therefore \stackrel{\frac{n}{n}}{\varrho}$ \\
\hline 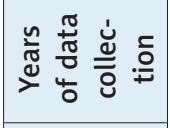 & 움 & 隹 \\
\hline 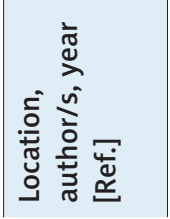 & 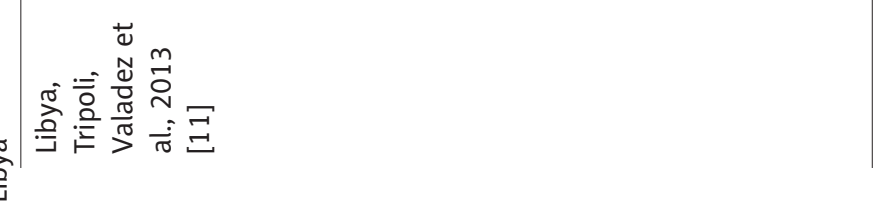 & 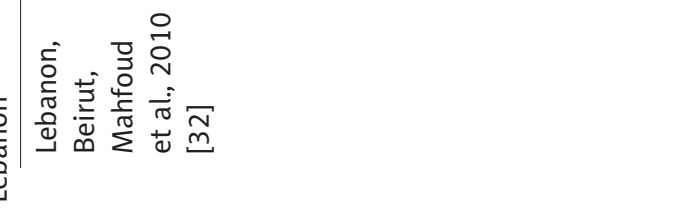 \\
\hline
\end{tabular}




\begin{tabular}{|c|c|c|}
\hline$=\stackrel{\widetilde{Q}}{0}$ & $\stackrel{m}{m}$ & $\stackrel{m}{m}$ \\
\hline 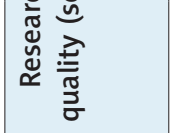 & 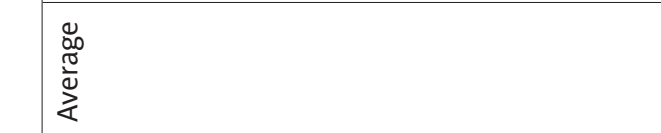 & 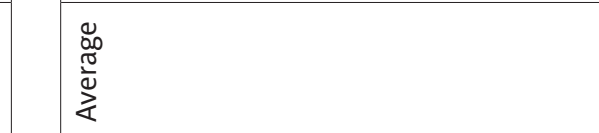 \\
\hline 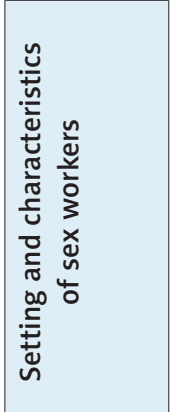 & 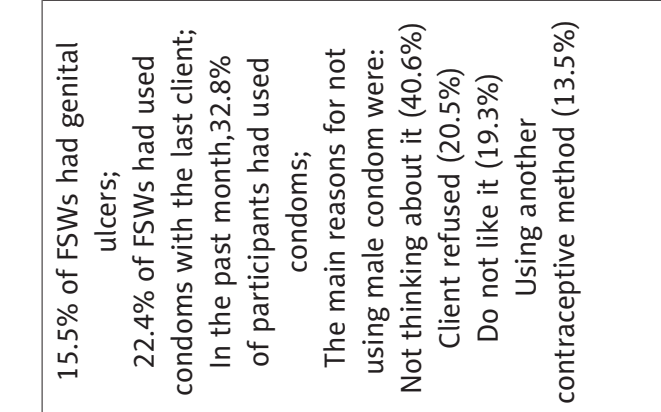 & 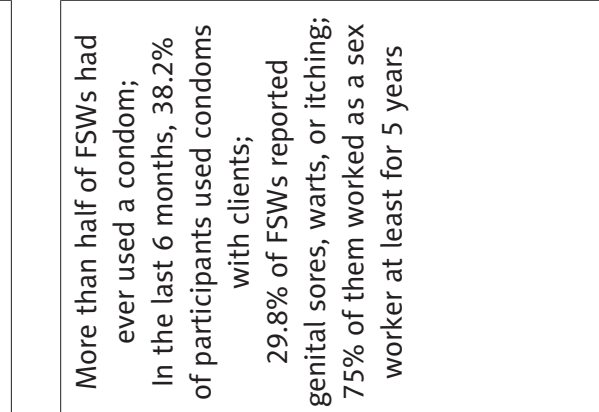 \\
\hline 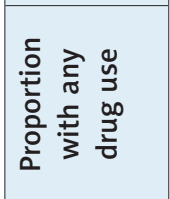 & 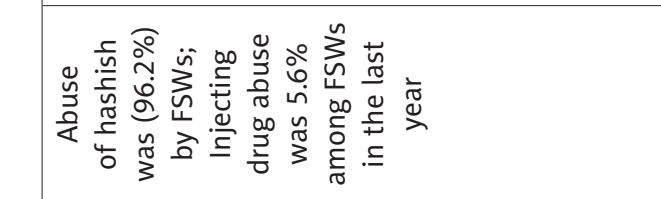 & 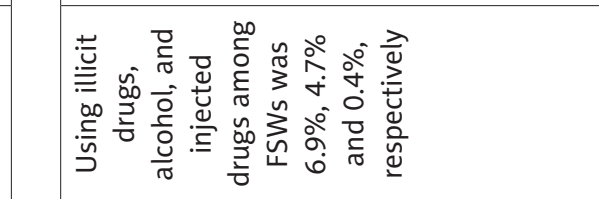 \\
\hline 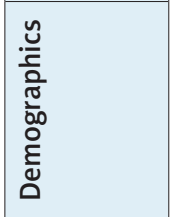 & 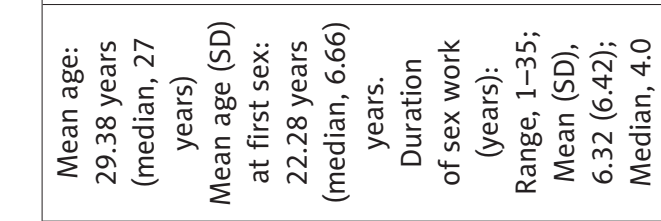 & 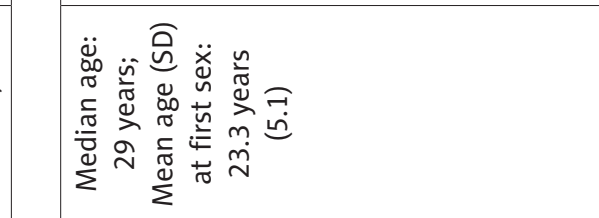 \\
\hline 》竞总 & 竞 & 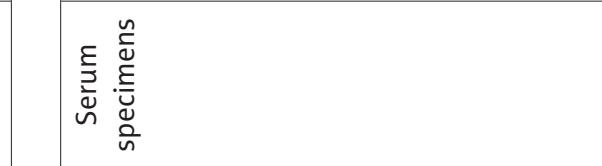 \\
\hline 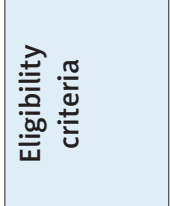 & 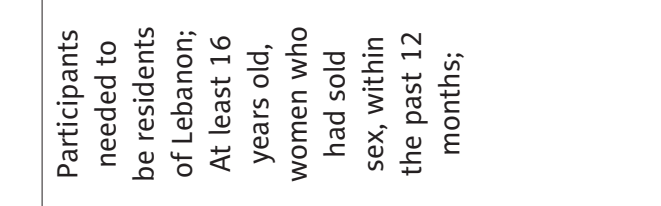 & 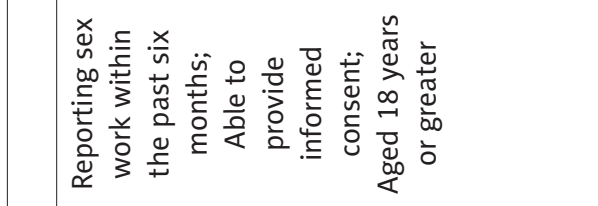 \\
\hline 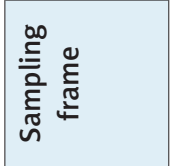 & 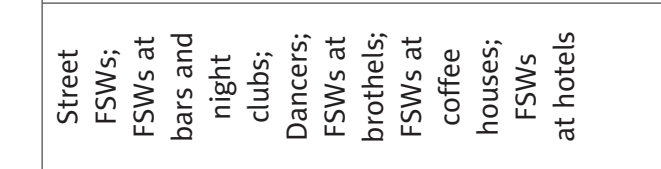 & 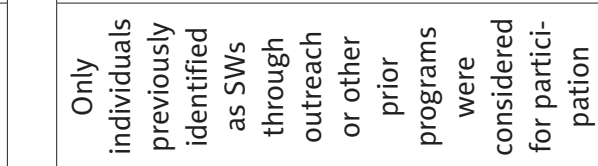 \\
\hline 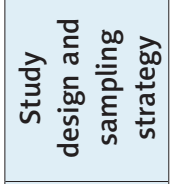 & 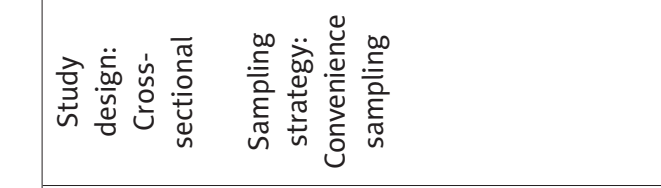 & 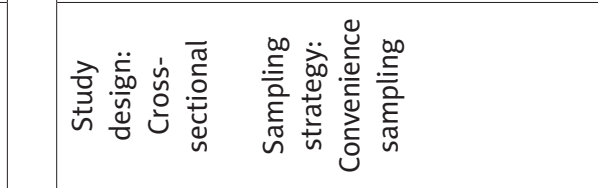 \\
\hline 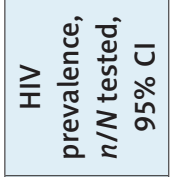 & ○े $\frac{\widehat{\partial}}{\tilde{y}}$ & 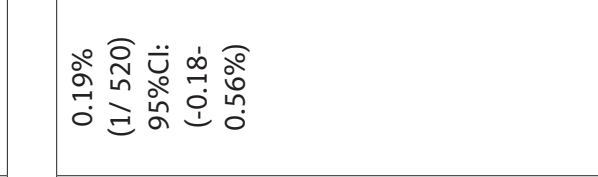 \\
\hline 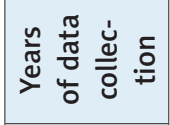 & 엉웅 & ठ் \\
\hline 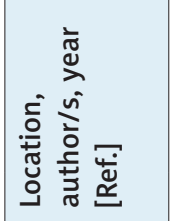 & 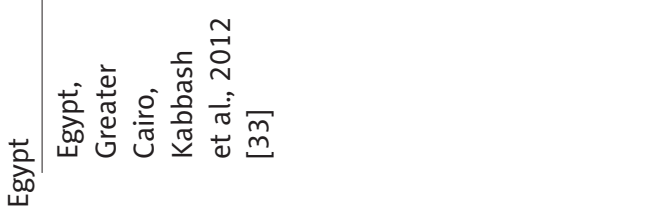 & 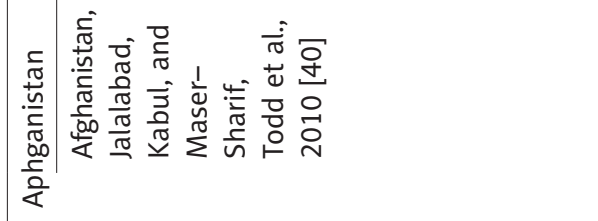 \\
\hline
\end{tabular}




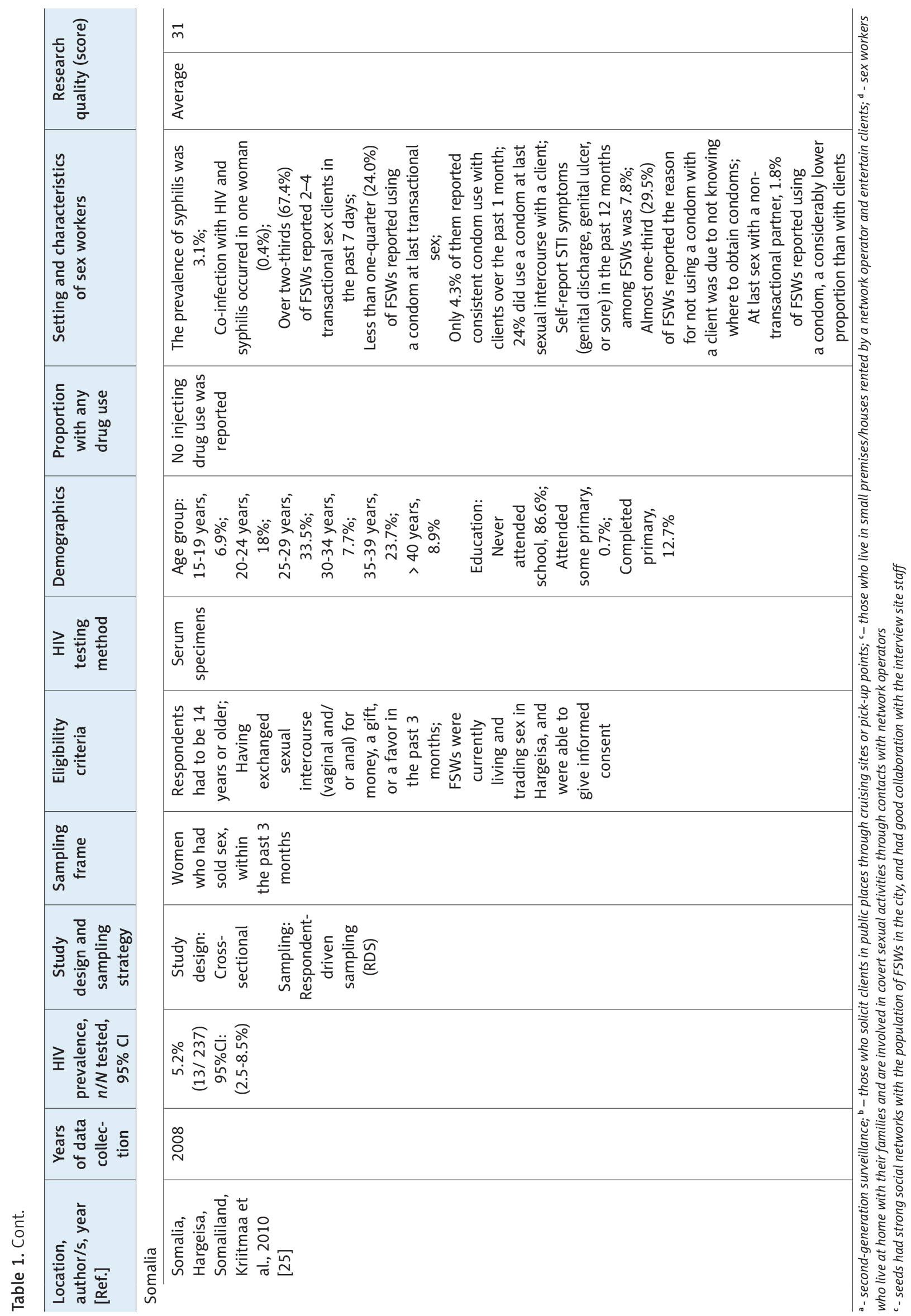


blood samples] and serum groups), and sample size categories (387 and lower, over 387). Stata version 11 (Stata Corp., College Station, TX, USA) was used to analyze the data.

\section{Results}

In this study, 156 papers were retrieved by searching the databases, conference abstracts, reference lists, and reports published by the WHO. Eventually, 21 papers were selected for the review (Figure 1). Table 1 shows the information extracted from these 21 papers, in which 17,205 FSWs were investigated.

The results of quality assessment revealed that $5(23.8 \%)$ studies were of good-quality [11, 13, 21-23], 11 (52.4\%) were of medium-quality [5, 24-33], and 5 (23.8\%) were of poorquality [34-38].

The study designs of 16 papers were cross-sectional [11, $22,24,25,27,28,31-40]$, and the rest of them were national surveys for AIDS [13, 21, 23, 26, 41]. Out of 21 Eastern Mediterranean countries, only 8 countries had conducted studies on the prevalence of HIV infection among FSWs. The majority of these studies were conducted in Iran [13, 23, 24, 26-28, 34, 35] and Pakistan [22, 29, 30, 36-38].

Moreover, 13 papers were exclusively about FSWs $[13,21$, 23-25, 27-29, 31, 33-35, 40], and the rest of them classified FSWs as a sub-group of the population under study [11, 32, $36-38,41]$. Nearly 16 papers had been published in the last 10 years $[11,13,21-24,26-28,30,33-35,38-40]$.

In 11 studies included in this systematic review, the average age of FSWs was 30 and lower [11, 21, 22, 24, 25, 31, 33,
38-41]. FSWs had different education levels, and more than a quarter of FSWs had elementary or junior high school education in 11 papers $[11,13,21,23,24,26,27,31,33,35,37]$. Moreover, in other studies, more than half of FSWs were illiterate $[22,25,30]$. The rest of the papers had not reported education levels [28, 34, 36, 38, 39].

The onset age of sexual contact had not been reported in 12 studies [5, 13, 21, 23, 24, 26, 30-36]. Studies reported that the mean age of FSWs at first sex was after 15 years [13, 23, $31,36]$, before 18 years [21, 34, 35], at 18 years [24], $22 \pm 5.6$ years [30], $23 \pm 5.1$ years [33, 40], or 25 years [26]. The average time, in which women worked as FSWs ranged from 2 to 6 years in five studies $[21,26,27,32,36,40]$. Some other papers reported the frequency of genital infectious diseases [11, $22,24,25,34-36,38]$, candidiasis and cervicitis [27], genital ulcers $[23,33,40]$, and pelvic inflammation [36] among FSWs. In several papers, FSWs never or rarely used condoms during sexual contact $[24,28,36]$. However, the consistency of using condoms among FSWs were as high as 65\% in Pakistan, 64\% in Lebanon, and $62 \%$ In Iran $[22,27,32]$. Condom use among regular and non-regular clients was reported to be respectively, $92 \%$ and $98 \%$ in Lebanon [32], and 63.4\% among non-regular clients in Libya [11] and 57.1\% in Iran [13]. A study from Sudan reported that $36 \%$ of FSWs used condoms for all clients [31], and in one study from Kerman, Iran, not using condoms was reported to be $17 \%$ and $22 \%$ with paying and non-paying partners, respectively [24]. Studies in Egypt and Sudan have mentioned that the main reasons for not using male condoms were not knowing where to obtain condoms [25], client refusal, not liking it, using another contraceptive method, not

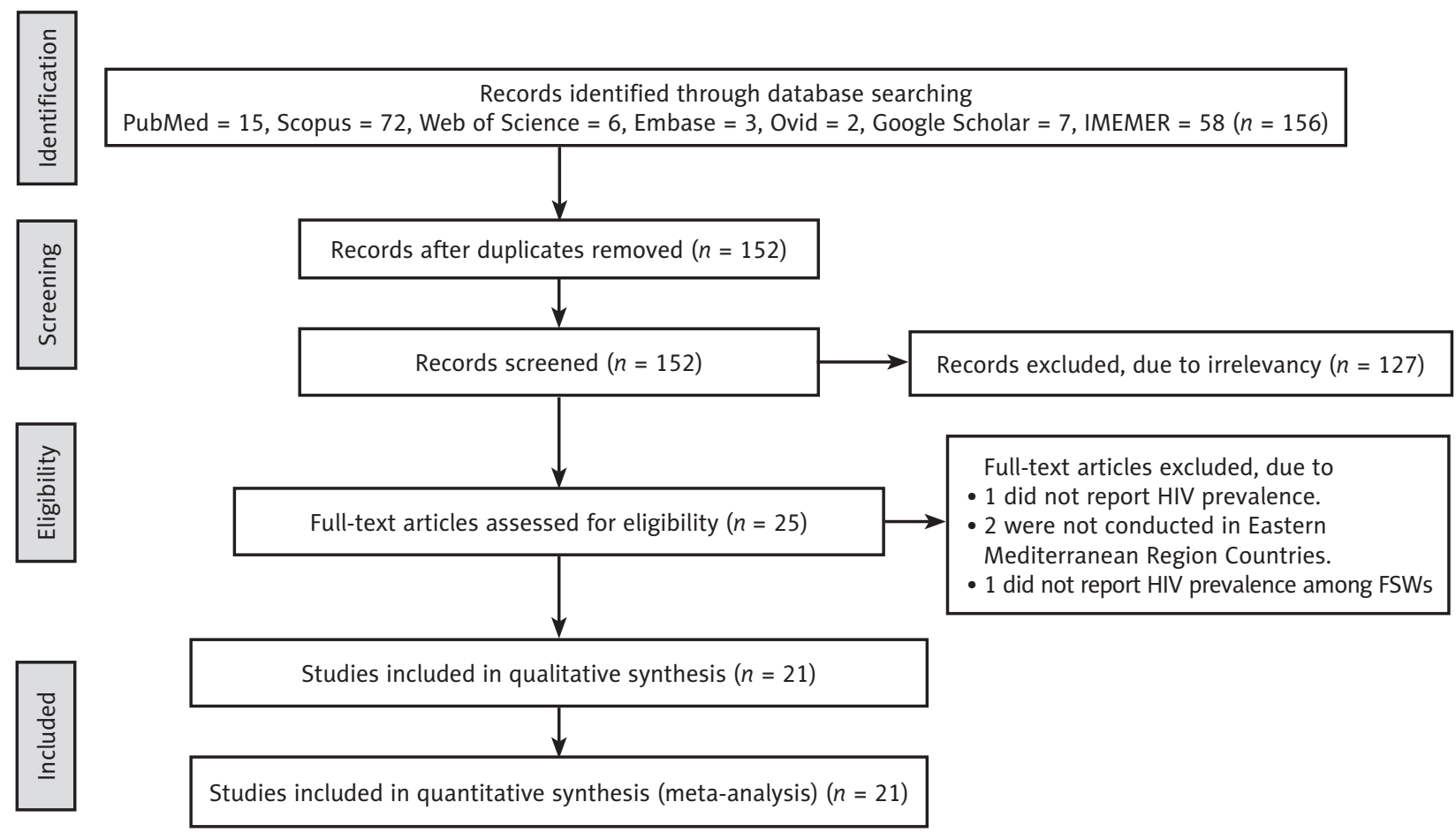

Figure 1. Flow diagram of the search strategy for systematic review on the prevalence of HIV among female sex workers (FSWs) in the Eastern Mediterranean Region by 2020 
Table 2. Egger's test for detection of publication bias for studies included in HIV infection among FSWs in the Eastern Mediterranean Region by 2020

\begin{tabular}{l|c|c|c|c|c}
\hline Std_Eff & Coef. & Std. error & $t$ & $P>|t|$ & $(95 \% \mathrm{Cl})$ \\
\hline Slope & -0.00015 & 0.002 & -0.07 & 0.945 & $(-0.00472,0.004)$ \\
\hline Bias & 3.38 & 0.73 & 4.61 & $<0.001$ & $(1.84,4.92)$ \\
\hline
\end{tabular}

Table.3. Meta-regression of some covariates on the prevalence of HIV infection among FSWs in the Eastern Mediterranean Region by 2020

\begin{tabular}{|c|c|c|c|c|c|}
\hline \multirow[t]{2}{*}{ Model } & \multicolumn{5}{|c|}{ Prevalence of HIV infection } \\
\hline & Coefficient $(95 \% \mathrm{CI})$ & $\tau^{2}$ & Adj. $R^{2}$ & $P^{2}$ & $p$-value \\
\hline No covariates & $0.025(0.0043-0.038)$ & 0.00059 & - & $89.99 \%$ & 0.001 \\
\hline \multicolumn{6}{|l|}{ Univariate } \\
\hline Sample size & $-5.89 \mathrm{e}-06(-0.0000173,5.49 \mathrm{e}-06)$ & 0.0006 & $-2.66 \%$ & $89.97 \%$ & 0.292 \\
\hline Years of data collection & $0.0028(-0.00015,0.0055)$ & 0.0004 & $27.06 \%$ & $87.43 \%$ & 0.04 \\
\hline All covariates & - & 0.000389 & $35.01 \%$ & $87.75 \%$ & \\
\hline Sample size & $-7.65 e-06(-0.0000172,1.86 e-06)$ & & & & 0.11 \\
\hline Years of data collection & $0.0239032(-0.0033577,-0.051164)$ & & & & 0.01 \\
\hline
\end{tabular}

thinking about it, and partner's refusal $[31,33]$. The most common reported reason for starting to work as FSWs was poverty and financial problems [22].

The prevalence of drug use was high in the included papers and it ranged from 5\% in Pakistan [30] and Sudan [21], to $91 \%$ in Iran [34] and $96.2 \%$ in Egypt [33]. The drugs abused included Iranian crack or heroin (in $16 \%$ ), opium with methamphetamine (in $16 \%$ ), methamphetamine (in 25\%), methamphetamine with alcohol (in $13 \%$ ) in Iran [27], and only alcohol in $4.7 \%$ [40], to $14 \%$ in Afghanistan and Iran, respectively [23]. In these studies, intravenous drug abuse ranged from $0.4 \%$ in Pakistan [22] and Afghanistan [40], to $38 \%$ in Iran [26]. One study in Iran reported that $11.6 \%$ of intravenous drug-addicted FSWs used non-sterilized syringes [13]. However, no statistics were reported on the use of drugs and alcohol in three other studies $[11,25,32]$.

In all the studies, laboratory methods were used to identify HIV infections, and six studies used DBS [13, 26, 29, 31, $34,41]$. The rest of the papers used direct blood sampling methods to diagnose HIV infection.

The prevalence of HIV infection ranged from $0 \%$ to $16 \%$ in the 21 papers reviewed. The prevalence of HIV infection was zero in three papers published from Iran [24], Lebanon [32], and Egypt [33]. The results of Egger's test for the funnel plot were statistically significant $(p<0.001)$, which means that publication bias was likely to occur (Table 2).

Heterogeneity of the reported prevalence was very high among the studies $\left(I^{2}=89.9 \%, \mathrm{Q}=199.77, \mathrm{df}=11, p<0.001\right)$, and therefore a meta-analysis was not applicable to perform [42]. Moreover, the studies were plotted on Galbraith chart (Figure 2). Some study results were out of the $95 \% \mathrm{CI}$, and this showed high heterogeneity. It was neither reasonable
Table 4. Characteristics of sub-group analysis of the included studies on HIV infection among FSWs in the Eastern Mediterranean Region by 2020

\begin{tabular}{l|c|c|c}
\hline Sub-group & $\begin{array}{c}\text { Number } \\
\text { of studies }\end{array}$ & $I^{2}$ & $p$-value \\
\hline \begin{tabular}{l} 
Years of data collection \\
\hline$\leq 2010$
\end{tabular} & 13 & $86.24 \%$ & $<0.001$ \\
\hline$>2010$ & 8 & $90.70 \%$ & $<0.001$ \\
\hline \begin{tabular}{l} 
Laboratory method \\
\hline DBS
\end{tabular} & 6 & $92.17 \%$ & $<0.001$ \\
\hline Serum & 14 & $89.23 \%$ & $<0.001$ \\
\hline Sample size & 11 & $85.82 \%$ & $<0.001$ \\
\hline$\leq 387$ & 10 & $91.54 \%$ & $<0.001$ \\
\hline$>387$ &
\end{tabular}

nor logical to remove specific studies to make the remaining results homogeneous for a meta-analysis.

The result of meta-regression demonstrated that no significant relation was observed between the sample size and the prevalence of HIV, but there was a significant relation between the year of research and the prevalence of HIV (Table 3).

Authors used "visual binning" in SPSS v. 22, and divided the studies into sub-groups according to the years of data collection. The studies were divided into two groups (2010 and before, and after 2010), and in each category, heterogeneity was assessed. Heterogeneity in the 2010 and before group was $I^{2}=86.2 \%, \mathrm{Q}=87.23, \mathrm{df}=12, p \leq 0.001$, and in the after 2010 group, it was $I^{2}=90.7 \%, \mathrm{Q}=75.30, \mathrm{df}=7$, 
$p<0.001$ (Table 4). Therefore, meta-analysis was not done in these sub-groups because of high heterogeneity.

The studies were also divided according to the laboratory methods used, into DBS and serum groups. In the DBS method group, heterogeneity was high and $I^{2}=92.1 \%$, $\mathrm{Q}=63.85, \mathrm{df}=5$, and $p<0.001$. Heterogeneity in the serum method was also high and $I^{2}=89.23 \%, \mathrm{Q}=120.71, \mathrm{df}=13$, and $p<0.001$ (Table 4). Therefore, meta-analysis was not performed in these sub-groups due to high heterogeneity.

The studies were divided according to the sample size, into 387 and lower and more than 387. In the 387 and lower group, heterogeneity was high and $I^{2}=85.82 \%, \mathrm{Q}=70.53$, $\mathrm{df}=10$, and $p<0.001$. Heterogeneity in the more than 387 group was high and $I^{2}=91.54 \%, \mathrm{Q}=106.34$, $\mathrm{df}=9$, and $p<0.001$ (Table 4). Again, meta-analysis was not done in these sub-groups because of high heterogeneity.

\section{Discussion}

The reported prevalence of HIV infection varied differently in the studies on female sex workers in the EM countries, ranging from $0 \%$ to $16 \%$. The majority of FSWs had never or rarely used condoms during sexual contact. Also, genital infectious diseases, candidiasis, cervicitis, genital ulcers, warts, or itching and pelvic inflammation were reported among FSWs. The prevalence of drug use was high, and heroin, opium, methamphetamine, and alcohol were the drugs with the highest use among FSWs.

In the Eastern Mediterranean (EM) countries, only three countries, including South Sudan, Djibouti, and Somalia, have generalized epidemics; the remaining countries have an estimated HIV infection prevalence of below $1 \%$ in the general population, with either low-level epidemics or HIV infection epidemics concentrated in at-risk of HIV infection populations, and with limited spread to the general population [43].

Findings of our study showed that the prevalence rate of HIV infection among FSWs in the EM countries is different. Review studies in Europe (from 2005-2011) showed that there was a wide variation in HIV infection among FSWs at city level, and HIV infection prevalence among FSWs was highest in Eastern Europe in 2006, 2007, and 2009, within high HIV infection prevalence countries, such as Russia and Ukraine [12]. Also in the US (during 1984-2007), the prevalence of HIV infection among FSWs ranged from a minimum of $0.3 \%$ (1996-1998) to as high as $32.1 \%$ (19921994), and the pooled prevalence was $17.3 \%$ [42]. A review from Latin America and the Caribbean (from 1986 to 2010) showed that the median of HIV infection prevalence was 2.6\% (IQR: 0.6-4.2) among FSWs [15].

HIV infection surveys among FSWs in the eleven Eastern Mediterranean (EM) countries demonstrated that the prevalence rate was below $4 \%$ in all countries, with the exception of Somalia (5.2\%, 2007), Iran, and Djibouti [43]. The HIV infection prevalence among FSWs in Iran revealed an HIV infection prevalence of $4.5 \%$ in 2011 [13], whereas it was $2.6 \%$ in 2007; in Morocco, it was $2.7 \%$ in 2010 , and $19.7 \%$ in 2007 and $15.4 \%$ in 2009 in Djibouti [44]. However, data

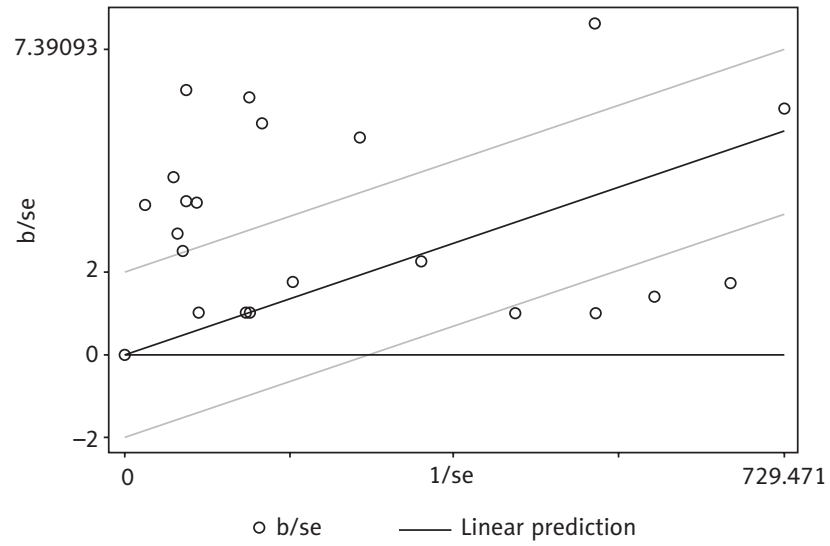

Figure 2. Galbraith plot for assessment of heterogeneity of articles on the prevalence of HIV among female sex workers (FSWs) in the Eastern Mediterranean Region by 2020

on HIV infection prevalence among FSWs is unavailable from several low- or high-income countries, including Bahrain, Iraq, Kuwait, Libya, Oman, Qatar, Saudi Arabia, South Sudan, Syria, and United Arab Emirates (UAE), and the existing evidence do not portray the real picture of HIV infection in this region $[43,44]$. On the other hand, HIV infection surveillance for evaluating HIV infection trends were performed in only four countries, included Djibouti, Iran, Morocco, and Pakistan. These countries have developed functioning HIV infection surveillance programs with national-level coverage. However, in the other 10 countries, HIV infection surveillance has been insufficient [43]. A review from the Middle East and North Africa showed a very low prevalence of HIV infection among FSWs in Afghanistan, Egypt, Jordan, Lebanon, Pakistan, Tunisia, and Yemen [45]. Despite the overall relatively low prevalence of HIV infection among FSWs, commercial heterosexual sex networks appear to be a cause of HIV infections in some countries, probably because of the larger size of these networks compared with other high-risk groups, such as homosexual men [45]. For example, in Morocco, commercial heterosexual sex networks contributed to about half of the HIV infection incidences, despite a rather low prevalence (2\%) of HIV infection among FSWs [21].

Studies have demonstrated the significance of sex work in the ongoing transmission of HIV infection and other sexually transmitted infections (STIs). In the EM countries, genital infectious diseases, candidiasis, cervicitis, genital ulcers, warts or itching, and pelvic inflammation were reported among FSWs. A review study done in Europe indicated that the prevalence of gonorrhea was reported to be $5 \%$ or less across the region in FSWs, with the exception of Georgia (12-18\%), and the prevalence of chlamydia was just over $20 \%$ in 2004-2006 [12]. Another review study from China reported that the prevalence of active syphilis ranged from $0.8 \%$ to $12.5 \%$, herpes from $29.7 \%$ to $70.8 \%$, chlamydia from $3.9 \%$ to $58.6 \%$, gonorrhea from $2.0 \%$ to $85.4 \%$, and trichomoniasis from $7.1 \%$ to $43.2 \%$ among FSWs [46]. 
Our review showed that the usage of condoms during sexual contact among FSWs was rare, and condom use among regular and non-regular clients was different. Several studies in African countries [47-49], China [50,51], various Asian countries [52,53], Latin America [54,55], and Europe [56] have shown that FSWs use condoms less frequently with their steady sex partners than with other clients [57].

The studies included in this review demonstrated that the prevalence of drug use among FSWs was high. A review showed that in Iran, over $70 \%$ of FSWs had ever used drugs, and around $15 \%$ had ever injected drugs. Moreover, older age, longer duration ( $>5$ years) of involvement in sex work, and a history of alcohol drinking were associated with a lifetime history of drug injection among FSWs [58]. In Iran, about $15 \%$ of FSWs reported injecting drugs, and these were over three times more likely to be infected with HIV compared with FSWs who never injected drugs [13].

It is possible that most HIV infections among FSWs in Iran are due to drug injection rather than sexual transmission [45]. Drug injection was the main cause of HIV infection among female sex workers in Europe, and the prevalence of HIV infection was higher among FSWs intravenous drug abusers. The prevalence of syphilis was highest among samples taken from FSWs in Eastern Europe [42].

Putting FSWs under surveillance is critical because their behaviors are usually considered illicit, and they are frequently stigmatized, deprived from health services, and exposed to sexual diseases and violence [12]. Between 1989 and 2007, less than 1 percent of HIV infection tests performed in the region were for these key populations [59]. In the EM countries, condoms are unpopular and rarely used. Reasons for such low use are the fact that some men do not believe they are at risk of STIs or HIV infection, they do not like the feeling of condoms, and FSWs are worried about their clients reactions to their suggestion of using a condom. Some believe condoms may be hazardous to use, some are embarrassed about buying and using condoms, or lacking the skills to use them [60].

Some of the essential ways of controlling HIV infection and AIDS include raising awareness in the society through training, consulting, and changing behaviors [61]. According to the WHO, the only effective way for controlling AIDS is training, especially among vulnerable groups [62]. Therefore, comprehensive knowledge about HIV infection and AIDS as well as using condoms should be seriously and actively provided to FSWs [63]. Studies have also shown that health training plans designed according to health belief models, can be effective in preventing AIDS $[62,64]$. One of the other educational methods for behavior change is theory of planned behavior (TPB), which considers intention as the main determinant of behavior. Studies have suggested that TPB can be used in education programs for HIV prevention [65], and it is necessary to provide free education and condoms to FSWs [62, 64].

In Tunisia, some forms of commercial sex are legal, and condoms are made available within regulated establishments. Around half of female sex workers in Morocco and Tunisia reported using a condom with their most recent client. However, in general, condom use is still largely tabooed in the region [59]. Four countries, including Pakistan, Egypt, Lebanon, and Tunisia, have provided HIV infection prevalence estimates for FSWs [66]. Different harm reduction programs, such as consistent condom use during high-risk sex and providing clean syringes, have been implemented in many EM countries, such as Egypt, Morocco, Tunisia, Bahrain, Iran, Lebanon, and Oman [66]. In Egypt, harm reduction interventions targeting PWID (people who inject drugs) and FSWs were established in 2008, and included education about safe sex and safe injection, HIV infection counseling, testing using rapid test kits, medical services for the management of sexually transmitted infections, and distribution of behavior-change booklets and brochures, needles, and condoms free of charge [67].

MENA region has had the lowest rate of antiretroviral treatment (ART) globally (11\%) [68]. In the UNAIDS 2017 and global AIDS update reports in 2019 [69], none of these countries reported using ART for FSWs. Although some countries, such as Bahrain, Djibouti, Iran, Egypt, and Tunisia, have used ART for patients based on the latest WHO guidelines, which are CD4+ counts under 500 cells $/ \mathrm{mm}^{3}$; but Iraq, Qatar, and Yemen did not report ART use [70].

There was a high-rate of heterogeneity among the papers included in this study. The difference in studies might be due to the difference in sampling, demographic characteristics of the participants, place of study, type of risky behaviors, and application of different laboratory kits for HIV diagnosis. Previous reviews have also reported high heterogeneity [6, 42]. In this review, the included papers, except for one paper from Pakistan [36], were conducted between 2004 to 2016. During this time period, different methods might have been used to investigate the prevalence. However, meta-regression showed no relation between the prevalence and the year of research.

Most of the studies included in this review were conducted in Iran (8 papers) $[13,23,24,26-28,34,35]$, Pakistan (6 papers) [22, 29, 36-38, 41], and Sudan (2 papers) [21, 31]. There was only one study conducted on the prevalence of HIV infection among FSWs in Afghanistan, Egypt, Lebanon, Libya, and Somalia. In the other 13 countries of the EM, such as Bahrain, Djibouti, Iraq, Jordan, Kuwait, Morocco, Palestine, Oman, Saudi Arabia, Syria, Tunisia, UAE, and Qatar, no studies were conducted. However, it is necessary to survey female sex workers in these countries in regard to HIV infection, risky behaviors, problems in performing diagnostic tests, antiretroviral therapy coverage, and HIV prevention programs.

The present review had several limitations. First, the included studies were different in regard to sample size, study implementation dates, and sampling strategies. The prevalence rates reported from Pakistan, Afghanistan, and Lebanon were from before 2008, but the prevalence rates reported for Iran and Libya were after 2010. Moreover, the high heterogeneity of these studies excluded undertaking a pooled analysis. Also, it was not possible to evaluate geographical diversity due to small number of studies and lack of information from countries, such as Bahrain, Iraq, Jordan, Kuwait, Qatar, Saudi Arabia, Oman, Syria, Tunisia, UAE, Yemen, Morocco, and Djibouti. National studies were only conducted in a few coun- 
tries of that region $[13,21,26,30,35,38]$, and other studies were limited to only some cities [11, 22-25, 27-29, 31-34, 3638, 40]. Another limitation was that different HIV infection diagnosing tests had been used in the included studies, which makes the results even more incomparable. The specimens collected in the studies were different (plasma, serum, whole blood, DBS, and oral fluid), and this depended on the logistics, populations, sites, HIV infection testing strategies, and algorithms. In this review, the majority of tests were performed based on serum and DBS samples.

\section{Acknowledgments}

We would like to thank Prof. Maryam Okhovati, who help us in searching the databases.

\section{Conflict of interest}

The authors declare no conflict of interest with respect to the research, authorship, and/or publication of this article.

\section{References}

1. United Nations Programme on HIV/AIDs. Global AIDS update 2016 [Internet]. World Health Organization. Geneva, Switzerland; 2016. Available from: http://www.unaids.org/sites/default/files/media_asset/UNAIDS_Gap_report_en.pdf.

2. United Nations of AIDS. Global AIDS update. Vol. 13. World Health Forum, Geneva 1992

3. Azizi H, Saboory E, Ghaderi S. The study of prostitute women's knowledge about ways of HIV transmission in Tehran in 1390. J Nurs Midwifery Urmia Univ Med Sci [Internet] 2014; 11. Available from: http://unmf.umsu.ac.ir/article-1-1646-en.html.

4. Baral S, Beyrer C, Muessig K, et al. Burden of HIV among female sex workers in low-income and middle-income countries: a systematic review and meta-analysis. Lancet Infect Dis 2012; 12: 538-549.

5. Baral S, Todd CSCS, Aumakhan B, Lloyd J, Delegchoimbol A, Sabin K. HIV among female sex workers in the Central Asian Republics, Afghanistan, and Mongolia: contexts and convergence with drug use. Drug Alcohol Depend 2013; 132 Suppl 1: S13-16.

6. Beyrer C, Crago AL, Bekker LG, et al. An action agenda for HIV and sex workers. Lancet 2014; 385: 287-301.

7. Abu-Raddad LJ, Ghanem KG, Feizzadeh A, Setayesh H, Calleja JMG, Riedner G. HIV and other sexually transmitted infection research in the Middle East and North Africa: promising progress? Sex Transm Infect 2013; 89 Suppl 3: iii1-4.

8. World Health Organization. New WHO guidelines to better prevent HIV in sex workers [Internet]. 2012. Available from: http:// www.who.int/hiv/mediacentre/feature_story/sti_guidelines/en/.

9. Kouyoumjian SP, Mumtaz GR, Hilmi N, et al. The epidemiology of HIV infection in Morocco: systematic review and data synthesis. Int J STD AIDS 2013; 24: 507-516.

10. Inciardi JA, Surratt HL, Kurtz SP, Weaver JC. The effect of serostatus on HIV risk behaviour change among women sex workers in Miami, Florida. AIDS Care 2005; 17 (Suppl 1): S88-101.

11. Valadez JJ, Berendes S, Jeffery C, et al. Filling the knowledge gap: measuring HIV prevalence and risk factors among men who have sex with men and female sex workers in Tripoli, Libya. PLoS One 2013; 8: e66701.

12. Platt L, Jolley E, Rhodes T, et al. Factors mediating HIV risk among female sex workers in Europe: a systematic review and ecological analysis. BMJ Open 2013; 3: e002836.
13. Sajadi L, Mirzazadeh A, Navadeh S, et al. HIV prevalence and related risk behaviours among female sex workers in Iran: results of the national biobehavioural survey, 2010. Sex Transm Infect 2013; 89 Suppl 3: iii37-40.

14. Rusakova M, Rakhmetova A, Strathdee SA. Why are sex workers who use substances at risk for HIV? Lancet 2015; 385: 211-212.

15. Miller W, Buckingham L, Sánchez-Domínguez M, Morales S, PazBailey G. Systematic review of HIV prevalence studies among key populations in Latin America and the Caribbean. Salud Publica Mex 2013; 55 Suppl 1: S65-78.

16. Arora P, Nagelkerke NJD, Jha P. A systematic review and metaanalysis of risk factors for sexual transmission of HIV in India. PLoS One 2012; 7: e44094.

17. Toska E, Pantelic M, Meinck F, Keck K, Haghighat R, Cluver L. Sex in the shadow of HIV: a systematic review of prevalence, risk factors, and interventions to reduce sexual risk-taking among HIV-positive adolescents and youth in sub-Saharan Africa. PLoS One 2017; 12: e0178106.

18. von Elm E, Altman DG, Egger M, Pocock SJ, Gøtzsche PC, Vandenbroucke JP. The strengthening the reporting of observational studies in epidemiology (STROBE) statement: guidelines for reporting observational studies. J Clin Epidemiol 2008; 61: 344-349.

19. Egger M, Davey Smith G, Schneider M, Minder C. Bias in metaanalysis detected by a simple, graphical test. BMJ 1997; 315: 629634.

20. Bax L, Ikeda N, Fukui N, Yaju Y, Tsuruta H, Moons K. More than numbers: the power of graphs in meta-analysis. Am J Epidemiol 2009; 169: 249-255.

21. Elhadi M, Elbadawi A, Abdelrahman S, et al. Integrated bio-behavioural HIV surveillance surveys among female sex workers in Sudan, 2011-2012. Sex Transm Infect 2013; 89 Suppl 3: iii17-22.

22. Khan MSMS, Unemo M, Zaman S, Lundborg CSCS. HIV, STI prevalence and risk behaviours among women selling sex in Lahore, Pakistan. BMC Infect Dis 2011; 11: 119.

23. Kazerooni PAP, Motazedian N, Motamedifar M, et al. The prevalence of human immunodeficiency virus and sexually transmitted infections among female sex workers in Shiraz, South of Iran: by respondent-driven sampling. Int J STD AIDS 2013; 25: 155-161.

24. Navadeh S, Mirzazadeh A, Mousavi L, Haghdoost AA, Fahimfar N, Sedaghat A. HIV, HSV2 and syphilis prevalence in female sex workers in Kerman, south-east Iran; using respondent-driven sampling. Iran J Public Health 2012; 41: 60-65.

25. Kriitmaa K, Testa A, Osman M, et al. HIV prevalence and characteristics of sex work among female sex workers in Hargeisa, Somaliland, Somalia. AIDS 2010; 24 Suppl 2: S61-67.

26. Mirzazadeh A, Nedjat S, Navadeh S, et al. HIV and related risk behaviors among female sex workers in Iran: bias-adjusted estimates from the 2010 national bio-behavoral survey. AIDS Behav 2014; 18 Suppl 1: S19-24.

27. Taghizadeh H, Taghizadeh F, Fathi M, Reihani P, Shirdel N, RezaeeS. Drug use and high-risk sexual behaviors of women at a drop-in center in Mazandaran Province, Iran, 2014. Iran J Psychiatry Behav Sci 2015; 9: e1047.

28. Karami M, Khazaei S, Poorolajal J, Soltanian A, Sajadipoor M. Estimating the population size of female sex worker population in Tehran, Iran: application of direct capture-recapture method. AIDS Behav 2017; 21: 2394-2400.

29. Altaf A, Agha A, Holte-McKinzie M, Abbas Q, Jafri SBSB, Emmanuel F. Size estimation, HIV prevalence and risk behaviours of female sex workers in Pakistan. J Pak Med Assoc 2012; 62: 551-557.

30. Emmanuel F, Salim M, Akhtar N, Arshad S, Reza TETE. Secondgeneration surveillance for HIV/AIDS in Pakistan: results from the $4^{\text {th }}$ round of integrated behavior and biological survey 2011-2012. Sex Transm Infect 2013; 89 Suppl 3: iii23-28.

31. Abdelrahim MS. HIV prevalence and risk behaviors of female sex workers in Khartoum, north Sudan. AIDS 2010; 24 Suppl 2: S55-60. 
32. Mahfoud Z, Afifi R, Ramia S, et al. HIV/AIDS among female sex workers, injecting drug users and men who have sex with men in Lebanon: results of the first biobehavioral surveys. AIDS 2010; 24 Suppl 2: S45-54.

33. Kabbash IA, Abdul-Rahman I, Shehata YA, Omar AAR. HIV infection and related risk behaviours among female sex workers in greater Cairo, Egypt. East Mediterr Health J 2012; 18: 920-927.

34. Moayedi-Nia S, Bayat Jozani Z, Esmaeeli Djavid G, et al. HIV, HCV, HBV, HSV, and syphilis prevalence among female sex workers in Tehran, Iran, by using respondent-driven sampling. AIDS Care 2016; 28: $487-490$

35. Shahesmaeili A, Karamouzian M, Shokoohi M, al. Symptom-based versus laboratory-based diagnosis of five sexually transmitted infections in female sex workers in Iran. AIDS Behav 2018; 22: 19-25.

36. Baqi S, Nabi N, Hasan SNSN, et al. HIV antibody seroprevalence and associated risk factors in sex workers, drug users, and prisoners in Sindh, Pakistan. J Acquir Immune Defic Syndr Hum Retrovirol 1998; 18: 73-79.

37. Bokhari A, Nizamani NMNM, Jackson DJDJ, et al. HIV risk in Karachi and Lahore, Pakistan: an emerging epidemic in injecting and commercial sex networks. Int J STD AIDS 2007; 18: 486-492.

38. Waheed U, Satti HSHS, Arshad M, Farooq A, Rauf A, Zaheer HAHA. Epidemiology of HIV/AIDS and syphilis among high risk groups in Pakistan. Pak J Zool 2017; 49: 1829-1834.

39. Altaf A, Zahidie A, Agha A. Comparing risk factors of HIV among hijra sex workers in Larkana and other cities of Pakistan: an analytical cross sectional study. BMC Public Health 2012; 12: 279.

40. Todd CSCS, Nasir A, Stanekzai MRMR, et al. HIV, hepatitis B, and hepatitis $\mathrm{C}$ prevalence and associated risk behaviors among female sex workers in three Afghan cities. AIDS 2010; 24 (Suppl 2): S69-75.

41. Emmanuel F, Thompson LHLH, Salim M, et al. The size and distribution of key populations at greater risk of HIV in Pakistan: implications for resource allocation for scaling up HIV prevention programmes. Sex Transm Infect 2013; 89 (Suppl 2): ii11-17.

42. Paz-Bailey G, Noble M, Salo K, Tregear SJ. Prevalence of HIV among U.S. female sex workers: systematic review and meta-analysis. AIDS Behav 2016; 20: 2318-2331.

43. Bozicevic I, Riedner G, Calleja JMG. HIV surveillance in MENA: recent developments and results. Sex Transm Infect 2013; 89 (Suppl 3): iii11-6.

44. Karamouzian M, Madani N, Doroudi F, Haghdoost AA. Improving the quality and quantity of HIV data in the middle east and North Africa: key challenges and ways forward. Int J Health Policy Manag 2017; 6: 65-69.

45. Mumtaz GR, Riedner G, Abu-Raddad LJ. The emerging face of the HIV epidemic in the Middle East and North Africa. Curr Opin HIV AIDS 2014; 9: 183-191.

46. Poon A, Li Z, Wang N, Hong Y. Review of HIV and other sexually transmitted infections among female sex workers in China. AIDS Care 2011; 23 Suppl 1: 5-25.

47. Stoebenau K, Hindin M, Nathanson C, Rakotoarison P, Razafintsalama V. “... But then he became my sipa”: the implications of relationship fluidity for condom use among women sex workers in Antananarivo, Madagascar. Am J Public Health 2009; 99: 811-819.

48. Kayembe P, Mapatano M, Busangu AF, et al. Determinants of consistent condom use among female commercial sex workers in the Democratic Republic of Congo: implications for interventions. Sex Transm Infect 2008; 84: 202-206.

49. Voeten HACM, Egesah OB, Varkevisser CM, Habbema JDF. Female sex workers and unsafe sex in urban and rural Nyanza, Kenya: regular partners may contribute more to HIV transmission than clients. Trop Med Int Heal 2007; 12: 174-182.

50. Lau JTF, Ho SPY, Yang X, Wong E, Tsui HY, Ho KM. Prevalence of HIV and factors associated with risk behaviours among Chinese female sex workers in Hong Kong. AIDS Care 2007; 19: 721-732.
51. Zhao R, Wang B, Fang X, Li X, Stanton B. Condom use and self-efficacy among female sex workers with steady partners in China. AIDS Care 2008; 20: 782-790.

52. Tran TTM, Le CL, Nguyen TL. Factors associated with inconsistent condom use among female sex workers in Nha Trang, Vietnam. Asia Pac J Public Health 2008; 20: 370-378.

53. Sopheab H, Morineau G, Neal JJ, Saphonn V, Fylkesnes K. Sustained high prevalence of sexually transmitted infections among female sex workers in Cambodia: high turnover seriously challenges the $100 \%$ Condom Use Programme. BMC Infect Dis 2008; 8: 167.

54. Barrientos JE, Bozon M, Ortiz E, Arredondo A. HIV prevalence, AIDS knowledge, and condom use among female sex workers in Santiago, Chile. Cad Saude Publica 2007; 23: 1777-1784.

55. Gutiérrez JP, Molina Yépez D, Samuels F, Bertozzi FM. Inconsistent condom use among sexual workers in Ecuador: results from a behavior survey. Salud Publica Mex 2006; 48: 104-112.

56. van Veen MG, Götz HM, van Leeuwen PA, Prins M, van de Laar MJW. HIV and sexual risk behavior among commercial sex workers in the Netherlands. Arch Sex Behav 2010; 39: 714-723.

57. Ulibarri MD, Strathdee SA, Lozada R, et al. Condom use among female sex workers and their non-commercial partners: effects of a sexual risk intervention in two Mexican cities. Int J STD AIDS 2012; 23: 229-234.

58. Karamouzian M, Mirzazadeh A, Rawat A, et al. Injection drug use among female sex workers in Iran: findings from a nationwide bio-behavioural survey. Int J Drug Policy 2017; 44: 86-91.

59. Setayesh H, Roudi-Fahimi F, El Feki S, Ashford LS. HIV and AIDS in the Middle East and North Africa. Population Reference Bureau, Washington 2014.

60. KabbashIA,El-Sayed NM, Al-Nawawy AN, ShadyIK, Abou Zeid MS. Condom use among males (15-49 years) in lower Egypt: knowledge, attitudes and patterns of use. Eastern Mediterranean Health Journal 2007; 13: 1405-1416.

61. Bakhtiari S, Maleki Z, Alavi K, Ghoddousi J. Knowledge and attitudes of Tehran high schools (district 1) about HIV. J Dent Sch 2006; 26: 115-124.

62. Behzadpour M, Khanjani N. The prevalence of different human immunodeficiency virus transmission routes and knowledge about AIDS in infected people with HIV in Sirjan. JFUMS 2012; 2: 45-52.

63. Malery Khah Langeroudi Z, Rahimi Movaghar A, Delbarpour Ahmadi S, Esmaeili M. Barriers of condom use among female sex workers in Tehran, a qualitative study. SJSPH 2014; 12: 23-34.

64. Patrício ACF de A, Bezerra VP, Nogueira JA, Moreira MASP, Camargo BV, Santos J de S. Knowledge of women sex workers about HIV/AIDS. Int Arch Med 2016; 9. Available from: http://imed.pub/ ojs/index.php/iam/article/view/1479.

65. Sadeghi R, Khanjani N. Impact of educational intervention based on theory of planned behavior (TPB) on the AIDS-preventive behavior among health volunteers. Iran J Health Educ Health Promot 2015; 3: 23-31.

66. Harm Reduction International. The global state of harm reduction. 2012. Available from: http://www.ihra.net/global-state-of-harmreduction-2012.

67. Oraby D. Harm reduction approach in Egypt: the insight of injecting drug users. Harm Reduct J 2013; 10: 17.

68. United Nations Programme on HIV/AIDS (UNAIDS). UNAIDS data 2017 [Internet]. 2017. Available from: https://www.unaids. org/sites/default/files/media_asset/20170720_Data_book_2017_ en.pdf.

69. Global AIDS update 2019. Communities at the centre defending rights breaking barriers reaching people with HIV services [Internet]. Urban renaissance? 2019. Available from: https://www.unaids.org/sites/ default/files/media_asset/2019-global-AIDS-update_en.pdf.

70. World Health Organization. Guidelines for using HIV testing technologies in surveillance; 2009. 
Appendix A. Search strategy details in each database by 2020

\begin{tabular}{|c|c|c|c|c|c|}
\hline $\begin{array}{l}\text { Name } \\
\text { of database }\end{array}$ & Search strategy & $\begin{array}{l}\text { Yielded } \\
\text { number } \\
\text { of } \\
\text { articles }\end{array}$ & $\begin{array}{l}\text { Number } \\
\text { of } \\
\text { duplicate } \\
\text { articles }\end{array}$ & $\begin{array}{l}\text { Number } \\
\text { of } \\
\text { screened } \\
\text { articles }\end{array}$ & $\begin{array}{c}\text { Number } \\
\text { of articles } \\
\text { assessed } \\
\text { for } \\
\text { eligibility } \\
\text { criteria }\end{array}$ \\
\hline PubMed & 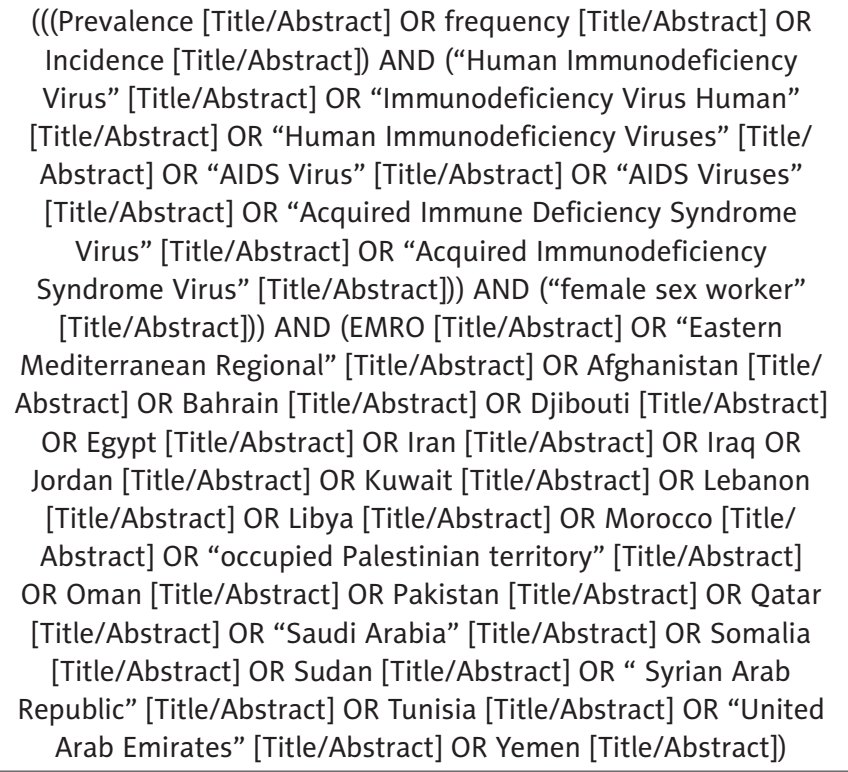 & 9 & 1 & 0 & 0 \\
\hline $\begin{array}{l}\text { Web } \\
\text { of Science }\end{array}$ & 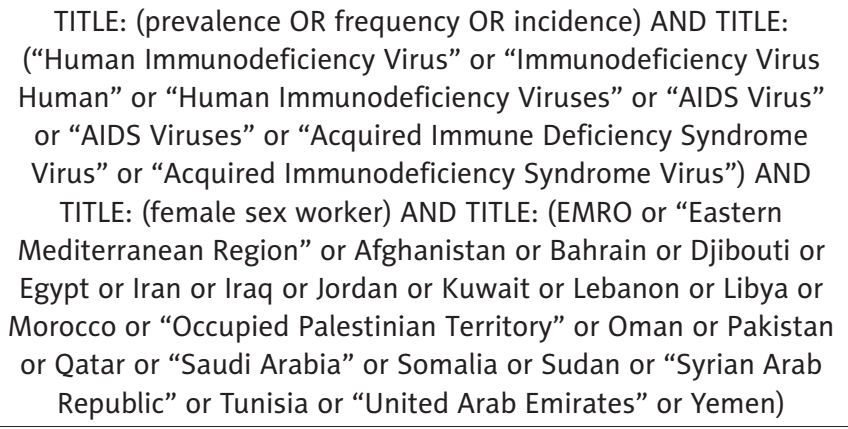 & 6 & 0 & 1 & 1 \\
\hline Scopus & $\begin{array}{l}\text { (TITLE-ABS-KEY (prevalence OR frequency OR incidence ) } \\
\text { AND TITLE-ABS-KEY (“Human Immunodeficiency Virus” OR } \\
\text { “Immunodeficiency Virus Human” OR “Human Immunodeficiency } \\
\text { Viruses” OR “AIDS Virus” OR “AIDS Viruses” OR “Acquired } \\
\text { Immune Deficiency Syndrome Virus" OR “Acquired } \\
\text { Immunodeficiency Syndrome Virus”) AND TITLE-ABS-KEY (female } \\
\text { AND sex AND worker) AND TITLE-ABS-KEY (emro OR “Eastern } \\
\text { Mediterranean Regional” OR afghanistan OR bahrain OR djibouti } \\
\text { OR egypt OR iran OR iraq OR jordan OR kuwait OR lebanon OR } \\
\text { libya OR morocco OR “occupied palestinian territory” OR oman } \\
\text { OR pakistan OR qatar OR “saudi arabia” OR somalia OR sudan) }\end{array}$ & 72 & 1 & 23 & 19 \\
\hline
\end{tabular}


Appendix A. Cont.

\begin{tabular}{|c|c|c|c|c|c|}
\hline $\begin{array}{l}\text { Name } \\
\text { of database }\end{array}$ & Search strategy & $\begin{array}{c}\text { Yielded } \\
\text { number } \\
\text { of } \\
\text { articles }\end{array}$ & $\begin{array}{l}\text { Number } \\
\text { of } \\
\text { duplicate } \\
\text { articles }\end{array}$ & $\begin{array}{l}\text { Number } \\
\text { of } \\
\text { screened } \\
\text { articles }\end{array}$ & $\begin{array}{l}\text { Number } \\
\text { of articles } \\
\text { assessed } \\
\text { for } \\
\text { eligibility } \\
\text { criteria }\end{array}$ \\
\hline Embase & 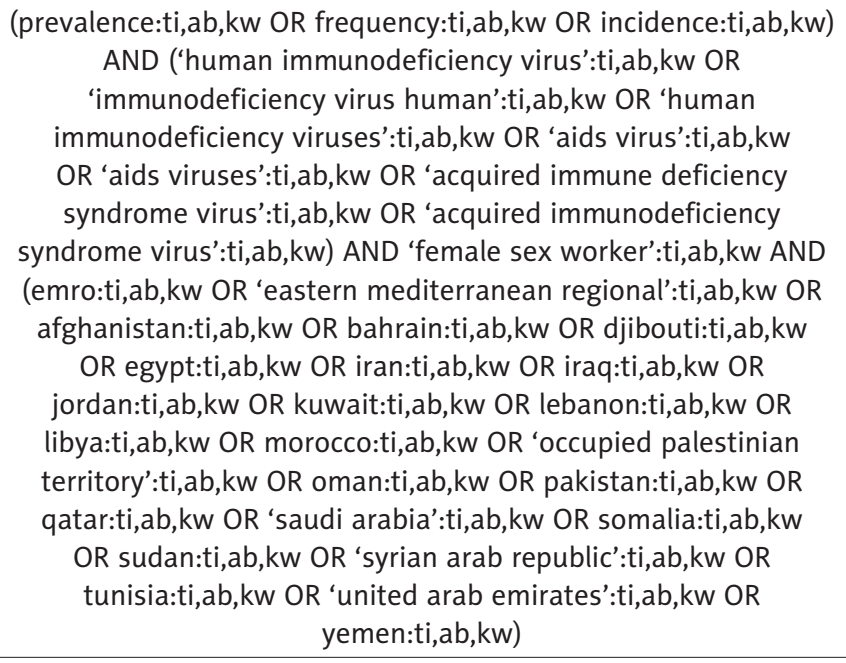 & 2 & 1 & 0 & 0 \\
\hline $\begin{array}{l}\text { Google } \\
\text { Scholar }\end{array}$ & $\begin{array}{l}\text { (Prevalence OR Frequency OR Incidence) AND (“Human } \\
\text { Immunodeficiency Virus" or "Immunodeficiency Virus Human" } \\
\text { or "Human Immunodeficiency Viruses" or "AIDS Virus" or "AIDS } \\
\text { Viruses" or "Acquired Immune Deficiency Syndrome Virus" or } \\
\text { "Acquired Immunodeficiency Syndrome Virus") AND “ female } \\
\text { sex worker" AND (EMRO or "Eastern Mediterranean Regional" } \\
\text { or Afghanistan or Bahrain or Djibouti or Egypt or Iran or Iraq or } \\
\text { Jordan or Kuwait or Lebanon or Libya or Morocco or "occupied } \\
\text { Palestinian territory" or Oman or Pakistan or Qatar or "Saudi } \\
\text { Arabia" or Somalia or Sudan or "Syrian Arab Republic" or } \\
\text { Tunisia or "United Arab Emirates" or Yemen) }\end{array}$ & 7 & 0 & 0 & 0 \\
\hline Ovid & $\begin{array}{l}\text { (Prevalence OR Frequency OR Incidence) AND (“Human } \\
\text { Immunodeficiency Virus" or "Immunodeficiency Virus Human" } \\
\text { or "Human Immunodeficiency Viruses" or "AIDS Virus" or "AIDS } \\
\text { Viruses" or "Acquired Immune Deficiency Syndrome Virus" or } \\
\text { "Acquired Immunodeficiency Syndrome Virus") AND “ female } \\
\text { sex worker" AND (EMRO or "Eastern Mediterranean Regional" } \\
\text { or Afghanistan or Bahrain or Djibouti or Egypt or Iran or Iraq or } \\
\text { Jordan or Kuwait or Lebanon or Libya or Morocco or "occupied } \\
\text { Palestinian territory" or Oman or Pakistan or Qatar or "Saudi } \\
\text { Arabia" or Somalia or Sudan or "Syrian Arab Republic" or } \\
\text { Tunisia or "United Arab Emirates" or Yemen) }\end{array}$ & 2 & 0 & 1 & 1 \\
\hline $\begin{array}{l}\text { Index } \\
\text { Medicus for } \\
\text { the Eastern } \\
\text { Medi- } \\
\text { terranean } \\
\text { Region } \\
\text { (IMEMsR) }\end{array}$ & Female sex worker AND prevalence of HIV & 58 & 1 & 0 & 0 \\
\hline
\end{tabular}

